\title{
A curious example of triangulated-equivalent model categories which are not Quillen equivalent
}

\author{
DANIEL DUGGer \\ BROOKE SHIPLEY
}

\begin{abstract}
The paper gives a new proof that the model categories of stable modules for the rings $\mathbb{Z} / p^{2}$ and $\mathbb{Z} / p[\epsilon] /\left(\epsilon^{2}\right)$ are not Quillen equivalent. The proof uses homotopy endomorphism ring spectra. Our considerations lead to an example of two differential graded algebras which are derived equivalent but whose associated model categories of modules are not Quillen equivalent. As a bonus, we also obtain derived equivalent dgas with non-isomorphic $K$-theories.
\end{abstract}

$18 \mathrm{E} 30,18 \mathrm{~F} 25,55 \mathrm{U} 35$

\section{Introduction}

This paper examines two model categories $\mathcal{M}$ and $\mathcal{M}_{\epsilon}$, namely the stable module categories of the rings $\mathbb{Z} / p^{2}$ and $\mathbb{Z} / p[\epsilon] /\left(\epsilon^{2}\right)$. It is known from Schlichting [17] that $\mathcal{M}$ and $\mathcal{M}_{\epsilon}$ have equivalent homotopy categories, and that algebraic $K$-theory computations show that $\mathcal{M}$ and $\mathcal{M}_{\epsilon}$ are not Quillen equivalent. Even more, by ToënVezzosi [21] it follows that the simplicial localizations of $\mathcal{M}$ and $\mathcal{M}_{\epsilon}$ are not equivalent. The point of this paper is to explore the homotopy theory of $\mathcal{M}$ and $\mathcal{M}_{\epsilon}$ in more detail, and to give a more elementary proof that they are not Quillen equivalent. Our proof uses homotopy endomorphism spectra rather than algebraic $K$-theory. Differential graded algebras come into the picture in that the model categories $\mathcal{M}$ and $\mathcal{M}_{\epsilon}$ are Quillen equivalent to modules over certain dgas.

Throughout the paper we fix a prime $p$ and let $k=\mathbb{Z} / p$. We write $R=\mathbb{Z} / p^{2}$ and $R_{\epsilon}=k[\epsilon] /\left(\epsilon^{2}\right)$. Each of these is a Frobenius ring, in the sense that the injectives and projectives are the same. As explained in Hovey [11, Section 2.2], there is a model category structure on the category of $R$-modules (respectively, $R_{\epsilon}$-modules) where the cofibrations are the injections, the fibrations are the surjections, and the weak equivalences are the "stable homotopy equivalences". For the latter, recall that two maps $f, g: J \rightarrow K$ are said to be stably homotopic if their difference factors through a projective; and a stable homotopy equivalence is a map $h: J \rightarrow K$ for which there 
exists an $h^{\prime}: K \rightarrow J$ where the two composites are stably homotopic to the respective identities. We write $\operatorname{Stmod}(R)$ for this model category structure, and throughout the paper we write $\mathcal{M}=\operatorname{Stmod}(R)$ and $\mathcal{M}_{\epsilon}=\operatorname{Stmod}\left(R_{\epsilon}\right)$. These are stable model categories, in the sense that the suspension functors on the homotopy categories are self-equivalences.

It is easy to see that the homotopy categories $\operatorname{Ho}(\mathcal{M})$ and $\operatorname{Ho}\left(\mathcal{M}_{\epsilon}\right)$ are both equivalent to the category of $k$-vector spaces. Even more, the suspension functor on both categories is isomorphic to the identity, and so $\operatorname{Ho}(\mathcal{M})$ and $\operatorname{Ho}\left(\mathcal{M}_{\epsilon}\right)$ are equivalent as triangulated categories. In [17] Schlichting studied the Waldhausen $K$-theory of the finitely-generated (or compact) objects in each category, and observed that when $p>3$ they differ starting at $K_{4}$. Specifically, $K_{4}(\mathcal{M}) \cong \mathbb{Z} / p^{2}$ whereas $K_{4}\left(\mathcal{M}_{\epsilon}\right) \cong \mathbb{Z} / p \oplus \mathbb{Z} / p$. These computations follow from classical computations of the algebraic $K$-theory of $R$ and $R_{\epsilon}$ from Evens-Friedlander [8] and Aisbett-LluisPuebla-Snaith [1]; see also Remark 4.9. By arguments from Dugger-Shipley [4], this difference in $K$-theory groups implies that $\mathcal{M}$ and $\mathcal{M}_{\epsilon}$ are not Quillen equivalent. By [21, Corollary 1.4], it even implies that the simplicial localizations of $\mathcal{M}$ and $\mathcal{M}_{\epsilon}$ are not equivalent.

Now, $K_{4}$ is a fairly elaborate invariant and the computations in [8] and [1] are quite involved. Given that $\mathcal{M}$ and $\mathcal{M}_{\epsilon}$ are such simple model categories, it is natural to ask for a more down-to-earth explanation for why they are not Quillen equivalent. Our goal in this paper is to give such an explanation.

Before explaining more about how we ultimately differentiate $\mathcal{M}$ and $\mathcal{M}_{\epsilon}$, it seems worthwhile to point out further ways in which they are very similar. Every $R$-module decomposes (non-canonically) as $F \oplus V$ where $F$ is free and $V$ is a $k$-vector space (regarded as an $R$-module via the quotient map $R \rightarrow k$ ). Similarly, every $R_{\epsilon}$-module also decomposes as the direct sum of a free module and a $k$-vector space. In some sense the categories of $R$-modules and $R_{\epsilon}$-modules are close to being equivalent even without the model structure, the only difference being in the endomorphisms of the free module $R$ compared to the free module $R_{\epsilon}$. But free modules are contractible in $\mathcal{M}$ and $\mathcal{M}_{\epsilon}$ ! This might lead one to mistakenly suspect that $\mathcal{M}$ and $\mathcal{M}_{\epsilon}$ were Quillen equivalent.

It is well-known that the homotopy category only encodes "first-order" information in a model category. One place that encodes higher-order information is the homotopy function complexes defined by Dwyer-Kan (see Hirschhorn [10, Chapter 17]). It turns out that every homotopy function complex in $\mathcal{M}$ is weakly equivalent to the corresponding homotopy function complex in $\mathcal{M}_{\epsilon}$, though. This is because $\mathcal{M}$ and $\mathcal{M}_{\epsilon}$ are additive categories, and therefore their homotopy function complexes have 
models which are simplicial abelian groups-in other words, they are generalized Eilenberg-MacLane spaces. It follows that the only information in the homotopy type of these function complexes is in their homotopy groups, and such information is already in the homotopy category.

It seems clear that the difference between $\mathcal{M}$ and $\mathcal{M}_{\epsilon}$ has to come from some process which considers more than just the maps between two objects; perhaps it has something to do with composition of maps, rather than just looking at maps by themselves. This is the tack we take in the present paper.

In Dugger [3] it is shown that if $X$ is an object in a stable, combinatorial model category then there is a symmetric ring spectrum $\mathrm{hEnd}(X)$-well defined up to homotopycalled the homotopy endomorphism spectrum of $X$. It is proven in [3] that this ring spectrum is invariant under Quillen equivalence. In the present paper we first argue that any Quillen equivalence between $\mathcal{M}$ and $\mathcal{M}_{\epsilon}$ must take the object $k \in \mathcal{M}$ to something weakly equivalent to the object $k \in \mathcal{M}_{\epsilon}$. We then compute the two homotopy endomorphism spectra of $k$ (considered as an object of $\mathcal{M}$ and as an object of $\mathcal{M}_{\epsilon}$ ) and we prove that these are not weakly equivalent as ring spectra. This then proves that $\mathcal{M}$ and $\mathcal{M}_{\epsilon}$ are not Quillen equivalent; see Theorem 4.5. The important point here is that it is the ring structures on the two spectra which are not weakly equivalent- the difference cannot be detected just by looking at the underlying spectra. In particular, we show that the $\mathbb{Z} / p$ homology algebras of the homotopy endomorphism spectra are not isomorphic.

\subsection{Connections with differential graded algebras (dgas)}

In general, computing homotopy endomorphism ring spectra is a difficult problem. In our case it is easier because the two model categories $\mathcal{M}$ and $\mathcal{M}_{\epsilon}$ are additive model categories, as defined in Dugger-Shipley [6]. The homotopy endomorphism spectra therefore come to us as the Eilenberg-MacLane spectra associated to certain "homotopy endomorphism dgas" (investigated in [6]), and what we really do is compute these latter objects. Unfortunately, such dgas are not invariant under Quillen equivalence, which is why we have to work with ring spectra. This brings us to the question of topological equivalence of dgas - that is to say, the question of when two dgas give rise to weakly equivalent Eilenberg-MacLane ring spectra. Our task is to show that the dgas arising from $\mathcal{M}$ and $\mathcal{M}_{\epsilon}$ are not topologically equivalent, which we do in Proposition 4.7 by using some of the techniques from Dugger-Shipley [7].

There is another connection with dgas, which comes from homotopical tilting theory. Each of the model categories $\mathcal{M}$ and $\mathcal{M}_{\epsilon}$ is an additive, stable, combinatorial model category with a single compact generator (the object $k$, in both cases). Let $T$ and 
$T_{\epsilon}$ denote the homotopy endomorphism dgas of $k$ as computed in $\mathcal{M}$ and $\mathcal{M}_{\epsilon}$, respectively; see Theorem 3.5 and Corollary 4.4. By results from Dugger [3], DuggerShipley [6], Schwede-Shipley [19] and Shipley [20], it follows that $\mathcal{M}$ and $\mathcal{M}_{\epsilon}$ are Quillen equivalent to the model categories Mod- $T$ and Mod- $T_{\epsilon}$, respectively. In fact, in this case it is quite easy to construct the Quillen equivalences directly without referring to the cited work above.

We can rephrase what we know about $\mathcal{M}$ and $\mathcal{M}_{\epsilon}$ in terms of $T$ and $T_{\epsilon}$. The model categories of modules Mod- $T$ and Mod- $T_{\epsilon}$ have triangulated-equivalent homotopy categories but are not Quillen equivalent. It is interesting to contrast this with the simpler case of rings: in [4] it is shown that if $S$ and $S^{\prime}$ are two rings then the model categories $\mathcal{C} h_{S}$ and $\mathcal{C} h_{S^{\prime}}$ are Quillen equivalent if and only if they have triangulatedequivalent homotopy categories (that is, if and only if $S$ and $S^{\prime}$ are derived equivalent). So this result does not generalize from rings to dgas.

It also follows from Schlichting's $K$-theory computations and [4] that the $K$-theories of $T$ and $T_{\epsilon}$ are non-isomorphic for $p>3$; see Remark 4.9. Thus $T$ and $T_{\epsilon}$ are derived equivalent dgas which for $p>3$ have non-isomorphic $K$-theories. Again, it was proven in [4] that this cannot happen for ordinary rings: derived equivalent rings have isomorphic $K$-theory groups. So this is another result which does not generalize from rings to dgas.

\subsection{Diagram categories}

While our use of homotopy endomorphism spectra to differentiate $\mathcal{M}$ and $\mathcal{M}_{\epsilon}$ is more elementary than using algebraic $K$-theory, one could make the case that it is still not all that elementary. The basic question of what is different about the underlying "homotopy theory" represented in $\mathcal{M}$ and $\mathcal{M}_{\epsilon}$ is perhaps still not so clear.

A different approach to these issues is the following. For any small category $I$, one has model structures on the diagram categories $\mathcal{M}^{I}$ and $\mathcal{M}_{\epsilon}^{I}$ in which the weak equivalences and fibrations are objectwise. Since a Quillen equivalence between $\mathcal{M}$ and $\mathcal{M}_{\epsilon}$ would induce an equivalence of $\operatorname{Ho}\left(\mathcal{M}^{I}\right)$ and $\operatorname{Ho}\left(\mathcal{M}_{\epsilon}^{I}\right)$ for any $I$, we would only need to find an $I$ where these categories are not equivalent to give another proof that $\mathcal{M}$ and $\mathcal{M}_{\epsilon}$ are not Quillen equivalent. The hope is that by looking at diagram categories one could restructure higher-order information about $\mathcal{M}$ (resp $\mathcal{M}_{\epsilon}$ ) into first-order information about $\mathcal{M}^{I}\left(\operatorname{resp} \mathcal{M}_{\epsilon}^{I}\right)$. In fact, by Renaudin [16, Theorem 3.3.2], the system of homotopy categories of diagram categories (the so-called derivateur) determines a homotopy theory just as a model category does. So we know that the nonequivalence of $\mathcal{M}$ and $\mathcal{M}_{\epsilon}$ must be detected in some way by considering $\operatorname{Ho}\left(\mathcal{M}^{I}\right)$ and $\operatorname{Ho}\left(\mathcal{M}_{\epsilon}^{I}\right)$. 
It is easy to see that for all $I$ and all diagrams $D_{1}, D_{2} \in \mathcal{M}_{\epsilon}^{I}$, the group $\operatorname{Ho}\left(\mathcal{M}_{\epsilon}^{I}\right)\left(D_{1}, D_{2}\right)$ is a $\mathbb{Z} / p$-vector space (the additive structure comes from the fact that $\mathcal{M}_{\epsilon}^{I}$ is a stable model category); see Proposition 5.2. It is likewise true that for all $I$ and all diagrams $D_{1}, D_{2} \in \mathcal{M}^{I}$, the abelian group $\operatorname{Ho}\left(\mathcal{M}^{I}\right)\left(D_{1}, D_{2}\right)$ is killed by $p^{2}$. By analogy with what happens in the algebraic $K$-theory computations, one might hope to find a certain category $I$ and two diagrams $D_{1}$ and $D_{2}$ in $\mathcal{M}^{I}$ such that $\operatorname{Ho}\left(\mathcal{M}^{I}\right)\left(D_{1}, D_{2}\right)$ is not killed by $p$. This would prove that $\mathcal{M}$ and $\mathcal{M}_{\epsilon}$ are not Quillen equivalent.

So far we have not been able to find such an $I$, but we would like to suggest this as an intriguing open problem. Here are some simple results to get things started, which are proved as Proposition 5.3 and Proposition 6.10. (For terminology, see Section 5 and Section 6).

Proposition 1.3 Let $I$ be a small, direct Reedy category. Then for any two diagrams $D_{1}, D_{2} \in \mathcal{M}^{I}$, the abelian group $\operatorname{Ho}\left(\mathcal{M}^{I}\right)\left(D_{1}, D_{2}\right)$ is a $\mathbb{Z} / p$-vector space.

Another thing one can prove is the following proposition.

Proposition 1.4 Let $I$ be a free category (or more generally, a category with $\mathbb{Z} / p-$ cohomological dimension equal to one). Then there is a bijection $\alpha: \operatorname{Ob} \operatorname{Ho}\left(\mathcal{M}^{I}\right) \rightarrow$ $\operatorname{Ob} \operatorname{Ho}\left(\mathcal{M}_{\epsilon}^{I}\right)$, with the property that for any two diagrams $D_{1}, D_{2} \in \operatorname{Ho}\left(\mathcal{M}^{I}\right)$ the abelian groups

$$
\operatorname{Ho}\left(\mathcal{M}^{I}\right)\left(D_{1}, D_{2}\right) \text { and } \operatorname{Ho}\left(\mathcal{M}_{\epsilon}^{I}\right)\left(\alpha D_{1}, \alpha D_{2}\right)
$$

are $\mathbb{Z} / p$-vector spaces of the same dimension.

The above proposition is weaker than saying that $\operatorname{Ho}\left(\mathcal{M}^{I}\right)$ and $\operatorname{Ho}\left(\mathcal{M}_{\epsilon}^{I}\right)$ are equivalent as categories, but it makes it seem likely that this is indeed the case. The categories $0 \rightarrow 1 \rightarrow \cdots \rightarrow n$ of $n$ composable arrows are examples of free categories.

The simplest category which has $\mathbb{Z} / p$-cohomological dimension greater than one is the coequalizer category $I$ consisting of three objects

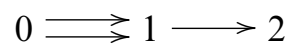

and four non-identity maps: the three shown above, and the map which is equal to the two composites. This is a directed Reedy category, so according to Proposition 1.3 all of the groups $\operatorname{Ho}\left(\mathcal{M}^{I}\right)\left(D_{1}, D_{2}\right)$ are $\mathbb{Z} / p$-vector spaces. We have been unable to detect any differences between $\operatorname{Ho}\left(\mathcal{M}^{I}\right)$ and $\operatorname{Ho}\left(\mathcal{M}_{\epsilon}^{I}\right)$ in this case. 
Remark 1.5 Another approach to detecting differences between $\mathcal{M}$ and $\mathcal{M}_{\epsilon}$ is mentioned in [14]. There Muro finds a difference in what he calls the "cohomologically triangulated structures" associated to $\mathcal{M}$ and $\mathcal{M}_{\epsilon}$, but only in the case $p=2$. See also Baues-Muro [2]. It seems likely that there is some connection between Muro's invariant and the one obtained in the present paper, although our invariant works at all primes.

\subsection{Acknowledgments}

The first author was partially supported by NSF grant DMS0604354. The second author was partially supported by NSF Grant DMS0706877 and the Centre de Recerca Matemàtica (Barcelona, Spain).

\section{Background on model categories of stable modules}

In this section we establish some basic facts about the categories $\mathcal{M}=\operatorname{Mod}-R$ and $\mathcal{M}_{\epsilon}=$ Mod- $R_{\epsilon}$ of $R$-modules and $R_{\epsilon}$-modules. We develop the results for $\mathcal{M}$, but then remark that the proofs all work identically for $\mathcal{M}_{\epsilon}$.

If $M$ is a module over $\mathbb{Z} / p^{2}$, let $\Gamma M$ denote $\left(\operatorname{Ann}_{M} p\right) / p M$. Note that this is a $\mathbb{Z} / p$-vector space. Let $C_{*}(M)$ denote the chain complex with $M$ in every dimension and where the differentials are all multiplication by $p$. So $\Gamma M$ is just the homology of $C_{*}(M)$, say in dimension 0 .

Lemma 2.1 Every module $M$ over $\mathbb{Z} / p^{2}$ is isomorphic (non-canonically) to a direct sum of $\Gamma M$ and a free module.

Proof Let $M$ be our module. Choose a $\mathbb{Z} / p$-basis $\left\{v_{i}\right\}$ for $p M$. For each $i$, there exists a $w_{i} \in M$ such that $p w_{i}=v_{i}$. Let $F$ be the submodule generated by the $w_{i}$. One readily checks that the $w_{i}$ are a free basis for $F$.

The inclusion $\mathrm{Ann}_{M} p \hookrightarrow M$ induces a map $\left(\operatorname{Ann}_{M} p\right) / p M \rightarrow M / F$. We claim this is an isomorphism. To see this, observe that we have a short exact sequence of chain complexes

$$
0 \rightarrow C_{*}(F) \rightarrow C_{*}(M) \rightarrow C_{*}(M / F) \rightarrow 0
$$

and $C_{*}(F)$ is exact, because $F$ is free. By the zig-zag lemma, one has $\Gamma(M) \cong$ $\Gamma(M / F)$. But on $M / F$ multiplication by $p$ is the zero map, since $F \supseteq p M$; so $\Gamma(M / F)=M / F$. 
Finally, as $M / F$ is a $\mathbb{Z} / p$-vector space we can choose a basis $\left\{\alpha_{j}\right\}$. Let $\pi: M \rightarrow$ $M / F$ be the quotient map. For any $j$, there exists a $\beta_{j} \in M$ such that $\pi\left(\beta_{j}\right)=\alpha_{j}$ and $p \beta_{j}=0$ (this is really just the zig-zag lemma again). This gives us a splitting for the exact sequence $0 \rightarrow F \hookrightarrow M \rightarrow M / F \rightarrow 0$ by sending $\alpha_{j}$ to $\beta_{j}$.

Remark 2.2 Note that by the above result $M \simeq \Gamma(M)$ in $\operatorname{Stmod}(R)$, since free modules are contractible.

Let $i$ : Vect $\hookrightarrow \mathcal{M}$ be the map which regards every vector space as an $R$-module via the projection $R \rightarrow k$. This is the inclusion of a full subcategory. Note that the composite $\Gamma \circ i$ is isomorphic to the identity.

It is easy to see that if $f: J \rightarrow K$ is a stable homotopy equivalence then $\Gamma(f)$ is an isomorphism (using that $\Gamma$ takes free modules to zero). So one has the diagram

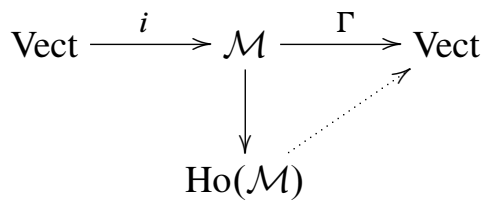

where the dotted arrow is the unique extension of $\Gamma$ (which we will also call $\Gamma$, by abuse). Since every object in $\operatorname{Ho}(\mathcal{M})$ is isomorphic to a $k$-vector space, it is clear that $\mathrm{Ho}(\mathcal{M}) \rightarrow$ Vect is bijective on isomorphism classes. It is also clear from the diagram that $\operatorname{Ho}(\mathcal{M}) \rightarrow$ Vect is surjective on hom-sets. We will prove below that it is actually an equivalence.

\subsection{Homotopies}

In model categories it is more common to deal with homotopies in terms of cylinder objects rather than path objects, as the former is more familiar. In stable module categories it seems to be easier to deal with path objects, however.

If $M$ is an $R$-module, let $F \rightarrow M$ be any surjection of a free module onto $M$. Write $P M=M \oplus F$. Let $i: M \hookrightarrow P M$ be the inclusion. Define $\pi: P M \rightarrow M \oplus M$ by having it be the diagonal on the first summand of $P M$, and on the second summand it is the composite $F \rightarrow M \hookrightarrow M \oplus M$, where the second map is the inclusion into the second factor. So the composite $M \rightarrow P M \rightarrow M \oplus M$ is the diagonal, $M \rightarrow P M$ is a trivial cofibration, and $P M \rightarrow M \oplus M$ is a fibration. Therefore $P M$ is a very good path object for $M$ in the sense of Quillen [15] and Hovey [11].

It follows that for any $R$-module $J$, the natural map

$$
\operatorname{coeq}(\mathcal{M}(J, P M) \rightrightarrows \mathcal{M}(J, M)) \rightarrow \operatorname{Ho}(\mathcal{M})(J, M)
$$


is an isomorphism. The following result is immediate.

Proposition 2.4 For any vector spaces $V$ and $W$ over $k$, the map $\operatorname{Vect}(V, W) \rightarrow$ $\operatorname{Ho}(\mathcal{M})(V, W)$ is an isomorphism.

Proof The two arrows $\mathcal{M}(V, P W) \rightrightarrows \mathcal{M}(V, W)$ are checked to be the same. The main point is that the only map $V \rightarrow W$ which factors through a free module is the zero map.

Corollary 2.5 The functors $i$ : $\operatorname{Vect} \rightarrow \operatorname{Ho}(\mathcal{M})$ and $\Gamma: \operatorname{Ho}(\mathcal{M}) \rightarrow$ Vect are an equivalence of categories.

For later use we record the following proposition.

Proposition 2.6 Every injection in $\mathcal{M}$ is isomorphic to a direct sum of injections of the following forms:

$$
0 \rightarrow k, \quad 0 \rightarrow R, \quad \text { id: } k \rightarrow k, \quad \text { id: } R \rightarrow R, \quad \text { and } \quad p: k \rightarrow R .
$$

Proof Let $j: M \hookrightarrow N$ be an injection of $R$-modules. We already know we can write $M \cong F \oplus V$ for some free module $F$ and some $k$-vector space $V$. So up to isomorphism we can assume $M=F \oplus V$, and that $M$ is a submodule of $N$. Consider the map of exact sequences

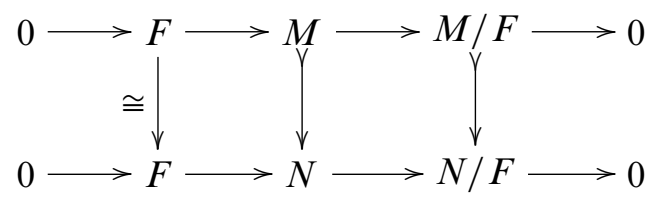

The evident projection $\pi: M \rightarrow F$ gives a splitting for the top exact sequence. Using that $F$ is injective, we can choose a map $N \rightarrow F$ whose restriction to $M$ is $\pi$. This gives a compatible splitting for the bottom exact sequence, showing that

$$
[M \stackrel{j}{\longrightarrow} N] \cong[F \longrightarrow F] \oplus[M / F \longrightarrow N / F] .
$$

The map id: $F \rightarrow F$ is isomorphic to a direct sum of maps id: $R \rightarrow R$. So now replacing $M$ with $M / F$ and $N$ with $N / F$, we can assume that the domain of $j$ is a $k$-vector space $V$.

So now assume $j$ is a map $V \rightarrow N$, where $V$ is a $k$-vector space. We again know that $N$ splits as $G \oplus W$ for some free module $G$ and some $k$-vector space $W$; so up to isomorphism we can assume $N=G \oplus W$ and that $V$ is a submodule of $N$. 
Consider the map of exact sequences

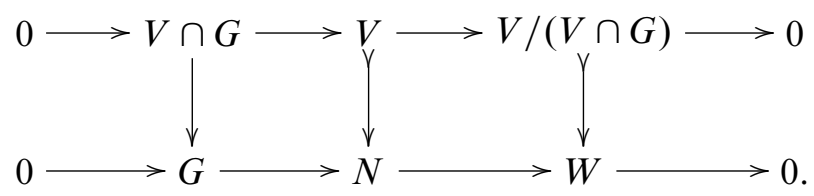

Since $V \cap G \hookrightarrow V$ is an inclusion of vector spaces, we can choose a splitting $\pi$. And then again using that $G$ is injective, we can choose a compatible splitting $N \rightarrow G$. So this shows

$$
[V \stackrel{j}{\longrightarrow} N] \cong[V \cap G \hookrightarrow G] \oplus[V /(V \cap G) \longrightarrow W] .
$$

The second map on the right is an inclusion of $k$-vector spaces, and so up to isomorphism it is a direct sum of maps id: $k \rightarrow k$ and $0 \rightarrow k$. So we are reduced to analyzing the first map on the right, which has the form $U \rightarrow G$ where $U$ is a $k$-vector space and $G$ is free.

Up to isomorphism we have that $U$ is a direct sum of the $k$. Using the inclusion $k \rightarrow R$ sending $1 \mapsto p$, we therefore obtain an embedding $U \hookrightarrow H$ where $H$ is a free module and the image of $U$ is $p H$. Since $G$ is injective, there is a map $H \rightarrow G$ extending $U \hookrightarrow G$. It is easy to see that $H \rightarrow G$ is also an injection.

So finally, consider the map of exact sequences

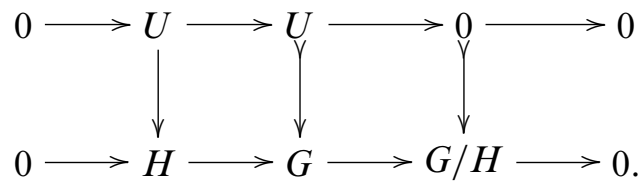

The bottom row is split (since $H$ is injective), and so there is a splitting $G / H \rightarrow G$ which is trivially compatible with the splitting $0 \rightarrow U$ of the top row. So this shows

$$
[U \rightarrow G] \cong[U \rightarrow H] \oplus[0 \rightarrow G / H]
$$

The first map on the right is isomorphic to a direct sum of maps $k \rightarrow R$ (by construction). Since $G / H$ is a direct summand of the free module $G$, it is itself free. So the second map on the right is isomorphic to a direct sum of maps $0 \rightarrow R$, and we are done.

\subsection{The case of $R_{\epsilon}$-modules}

All the results in the previous section have analogs for $\mathcal{M}_{\epsilon}$, and the proofs are essentially the same except replacing all occurrences of " $p$ " by " $\epsilon$ ". For instance, if $M$ is an $R_{\epsilon}-$ module then we define $\Gamma(M)=\left(\operatorname{Ann}_{M} \epsilon\right) / \epsilon M$. If anything, the proofs are slightly easier in the $\mathcal{M}_{\epsilon}$ case because every module is also a $k$-vector space. 


\subsection{Equivalences}

To say that two model categories $\mathcal{C}$ and $\mathcal{D}$ are Quillen equivalent means that there is a zig-zag

$$
\mathcal{C}=\mathcal{C}_{1} \stackrel{\sim}{\longrightarrow} \mathcal{C}_{2} \stackrel{\sim}{\longleftarrow} \mathcal{C}_{3} \stackrel{\sim}{\longrightarrow} \cdots \stackrel{\sim}{\longleftarrow} \mathcal{C}_{n}=\mathcal{D}
$$

of Quillen equivalences between $\mathcal{C}$ and $\mathcal{D}$. (Here we are regarding a Quillen pair $L: \mathcal{M} \rightleftarrows \mathcal{N}: R$ as a map of model categories in the direction of the left adjoint.) The derived functors of each Quillen equivalence induce an equivalence of the respective homotopy categories, and by composing these equivalences we obtain an equivalence $\operatorname{Ho}(\mathcal{C}) \simeq \operatorname{Ho}(\mathcal{D})$.

It is sometimes confusing to have $k$ denote both an $R$-module and an $R_{\epsilon}$-module. In these cases we will write $k_{\epsilon}$ to indicate $k$ thought of as an $R_{\epsilon}$-module.

Proposition 2.9 Suppose that one has a zig-zag of Quillen equivalences between $\mathcal{M}$ and $\mathcal{M}_{\epsilon}$. Then under the derived equivalence of homotopy categories, the object $k \in \operatorname{Ho}(\mathcal{M})$ maps to an object isomorphic to $k_{\epsilon} \in \operatorname{Ho}\left(\mathcal{M}_{\epsilon}\right)$.

Proof Recall that $\operatorname{Ho}(\mathcal{M})$ and $\operatorname{Ho}\left(\mathcal{M}_{\epsilon}\right)$ are both isomorphic to the category Vect of $k$-vector spaces. There is only one object (up to isomorphism) in this category whose set of endomorphisms has exactly $p$ elements.

\section{Stable module categories and differential graded modules}

One of our goals is to show that the model categories $\mathcal{M}$ and $\mathcal{M}_{\epsilon}$ are each Quillen equivalent to the model category of modules over certain dgas. In this section we set up the basic machinery for these Quillen equivalences, working in slightly greater generality.

Let $T$ be a Frobenius ring; a ring such that the projective and injective $T$-modules coincide. Consider $\operatorname{Stmod}(T)$, the stable model category on $T$-modules from [11, Theorem 2.2.12]. Here the cofibrations are the injections, the fibrations are the surjections, and the weak equivalences are the stable homotopy equivalences as described in the introduction. For two $T$-modules $M$ and $N$, denote by $[M, N]$ the stable homotopy classes of maps.

The goal of this section is to show that $\operatorname{Stmod}(T)$ is Quillen equivalent to a model category of $\mathrm{dg}$-modules over a dga if $\operatorname{Stmod}(T)$ has a compact, (weak) generator (see below). This extends to the model category level certain triangulated equivalences from Keller [12]. 
Definition 3.1 An object $M$ in $\operatorname{Stmod}(T)$ is compact if $\oplus_{i}\left[M, N_{i}\right] \longrightarrow\left[M, \oplus_{i} N_{i}\right]$ is an isomorphism, for every collection of objects $N_{i} . M$ is a (weak) generator if $[M, N]_{*}=0$ implies $N$ is weakly equivalent to 0 .

Lemma 3.2 If $M$ is stably equivalent to a finitely generated module, then $M$ is compact in $\operatorname{Stmod}(T)$.

Proof It is enough to check that every finitely-generated module is compact, and we leave this to the reader.

It follows from results of $[3 ; 6 ; 19 ; 20]$ that if an additive, stable, combinatorial model category has a compact weak generator then it is Quillen equivalent to the model category of modules over a dga (perhaps through a zig-zag of Quillen equivalences). Rather than invoke the heavy machinery from those sources, however, it is easier in the case of $\operatorname{Stmod}(T)$ to just establish the Quillen equivalence directly. We do this next.

Define the endomorphism dga associated to any object in $\operatorname{Stmod}(T)$ as follows. First, we need to fix projective covers and injective hulls for each $T$-module. To be specific we use the functorial cofibrant and fibrant replacements coming from the small object argument and the cofibrantly-generated model category structure [11, Theorem 2.1.14].

Definition 3.3 Define $I(M)$ by functorially factoring $M \longrightarrow 0$ as a composite $M \succ I(M) \stackrel{\sim}{\longrightarrow} 0$, a cofibration followed by a trivial fibration. Similarly, define $P(M)$ by functorially factoring $0 \longrightarrow M$ as $0 \stackrel{\sim}{\rightarrow} P(M) \rightarrow M$, a trival cofibration followed by a fibration.

Define $\Sigma M$ to be the cokernel of $M \longrightarrow I(M)$. Define $\Omega M$ to be the kernel of $P(M) \longrightarrow M$. Let $[M, N]_{*}$ be the graded stable homotopy classes of maps in $\operatorname{Ho}(\operatorname{Stmod}(T))$, so that $[M, N]_{n} \cong\left[\Sigma^{n} M, N\right] \cong\left[M, \Omega^{n} N\right]$.

To move from the setting of $T$-modules to differential graded modules we consider complete resolutions. A complete resolution of $M$ is an acyclic $\mathbb{Z}$-graded chain complex $P$ of projective (also injective) $T$-modules together with an isomorphism between $M$ and $Z_{-1} P$, the cycles of $P$ in degree -1 . Considering $M$ and $\Omega M$ as complexes concentrated in degree zero, observe that there is a canonical map of complexes $\pi: P \longrightarrow M$ obtained from the projection $P_{0} \rightarrow Z_{-1} P$. One can make a map of complexes $i: \Omega M \longrightarrow P$ by lifting $P(M) \rightarrow M$ to a map $P(M) \rightarrow P_{0}$, but this lifting is not canonical; however, the map $\Omega M \rightarrow P$ is canonical up to chain homotopy. 
One way to form a complete resolution is to take $P_{n}$ to be $I\left(\Sigma^{-(n+1)} M\right)$ for $n<0$ and for $n \geq 0$ to take $P_{n}$ to be $P\left(\Omega^{n} M\right)$ with the obvious differentials:

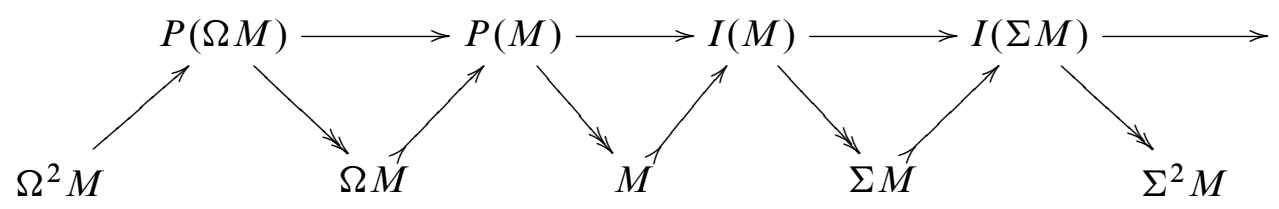

Denote this particular complete resolution by $P_{\bullet} M$.

Definition 3.4 Let $\mathcal{C} h_{T}$ be the category of $\mathbb{Z}$-graded chain complexes of $T$-modules. Given $X, Y$ in $\mathcal{C} h_{T}$ define $\operatorname{Hom}(X, Y)$ in $\mathcal{C} h_{\mathbb{Z}}$ as the complex with $\operatorname{Hom}(X, Y)_{n}=$ $\prod_{k} \operatorname{hom}_{T}\left(X_{k}, Y_{n+k}\right)$, the set of degree $n$ maps (ignoring the differentials). For $f=$ $\left(f_{k}\right) \in \operatorname{Hom}(X, Y)_{n}$ define $d f \in \operatorname{Hom}(X, Y)_{n-1}$ to be the tuple whose component in $\operatorname{hom}_{T}\left(X_{k}, Y_{n+k-1}\right)$ is $d_{Y} f_{k}+(-1)^{n+1} f_{k-1} d_{X}$. Notice that $\operatorname{Hom}(X, X)$ is a differential graded algebra.

We define $\mathcal{E}_{M}=\operatorname{Hom}\left(P \bullet M, P_{\bullet} M\right)$, the endomorphism dga of $M$. It follows from Lemma 3.6 below that $H_{*} \mathcal{E}_{M} \cong[M, M]_{*}$, the graded ring of stable homotopy classes of self maps of $M$. We denote by Mod- $\mathcal{E}_{M}$ the category of right differential graded modules over the dga $\mathcal{E}_{M}$. This has a model category structure where the weak equivalences are the quasi-isomorphisms and the fibrations are the surjections.

Note that if $N$ is a $T$-module then $\operatorname{Hom}\left(P_{\bullet} M, N\right)$ is a right module over $\mathcal{E}_{M}$.

Theorem 3.5 If $M$ is a compact, weak generator of $\operatorname{Stmod}(T)$ then there is a Quillen equivalence $\operatorname{Mod}-\mathcal{E}_{M} \rightarrow \operatorname{Stmod}(T)$ where the right adjoint is given by

$$
\operatorname{Hom}\left(P_{\bullet} M,-\right): \operatorname{Stmod}(T) \longrightarrow \operatorname{Mod}-\mathcal{E}_{M} \text {. }
$$

The proof of this result will be given below. We can better understand the adjoint functors in the Quillen equivalence by splitting the adjunction into two pieces:

$$
\operatorname{Mod}-\mathcal{E}_{M} \rightleftarrows \mathcal{C} h_{T} \underset{i_{0}}{\stackrel{c_{0}}{\rightleftarrows}} \operatorname{Stmod}(T) .
$$

In the first adjunction, the functors are just tensor and Hom: so the left adjoint sends a right $\mathcal{E}_{M}$-module $Q$ to $Q \otimes_{\mathcal{E}_{M}} P \bullet M$. In the second adjunction, the right adjoint $i_{0}$ sends a module $N$ to the chain complex with $N$ concentrated in degree 0 . So its left adjoint $c_{0}$ sends a chain complex $P$ to $P_{0} / \operatorname{im}\left(P_{1}\right)$. Thus, the left adjoint in our Quillen equivalence is the functor

$$
Q \mapsto c_{0}\left(Q \otimes_{\mathcal{E}_{M}} P \bullet M\right) .
$$


Note that this functor sends $\mathcal{E}_{M}$ to $M$.

We need the following statements to prove the theorem.

Lemma 3.6 Let $M$ and $N$ be $T$-modules and let $P$ be a complete resolution of $M$.

(a) There are isomorphisms $H_{k} \operatorname{Hom}(P, N) \cong[M, N]_{k}$, natural in $N$, for all $k \in \mathbb{Z}$.

(b) There are isomorphisms $H_{k} \operatorname{Hom}(N, P) \cong[N, \Omega M]_{k}$, natural in $N$, for all $k \in \mathbb{Z}$.

(c) The map $\pi_{*}: \operatorname{Hom}(P, P) \longrightarrow \operatorname{Hom}(P, M)$, induced by the map of complexes $\pi: P \rightarrow M$, is a quasi-isomorphism.

(d) The map $i_{*}: \operatorname{Hom}(P, P) \longrightarrow \operatorname{Hom}(\Omega M, P)$, induced by the map of complexes $i: \Omega M \rightarrow P$, is a quasi-isomorphism.

Proof We can lift the isomorphism $M \rightarrow Z_{-1} P$ to a map of complexes $P \bullet M \rightarrow P$. This gives a map $f: \Sigma^{k} M \rightarrow P_{-k} / \operatorname{im}\left(P_{-k+1}\right)$, which is a weak equivalence in $\operatorname{Stmod}(T)$. Any chain map $P \rightarrow N$ of degree $k$ induces a map $\Sigma^{k} M \rightarrow N$ by precomposition with $f$. This gives us a natural map $H_{k} \operatorname{Hom}(P, N) \rightarrow\left[\Sigma^{k} M, N\right]$.

Similarly, we can lift our isomorphism $Z_{-1} P \rightarrow M$ to a map $P \rightarrow P_{\bullet} M$, and this induces maps $Z_{k} P \rightarrow \Omega^{k+1} M$ which are again weak equivalences in $\operatorname{Stmod}(T)$. So any chain map $N \rightarrow P$ of degree $k$ induces a map $N \rightarrow Z_{k} P \rightarrow \Omega^{k+1} M$. This gives a natural map $H_{k} \operatorname{Hom}(N, P) \rightarrow\left[N, \Omega^{k+1} M\right]$.

It is a routine exercise to check that these two natural maps are isomorphisms.

For part (c), first recall that any map from a projective complex $Q$ to a bounded below acyclic complex $C$ is chain homotopic to zero (this follows from the Comparison Theorem of homological algebra). It follows that $\operatorname{Hom}(Q, C)$ is acyclic, since the cycles in degree $k$ are chain maps $\Sigma^{k} Q \rightarrow C$. Also, any map from an acyclic complex $C$ to a bounded above complex of injectives $I$ is chain homotopic to zero; so $\operatorname{Hom}(C, I)$ is acyclic.

Now we tackle (c). Let $F$ denote the kernel of the chain map $P \rightarrow M$, and consider the short exact sequence of complexes

$$
0 \rightarrow \operatorname{Hom}(P, F) \rightarrow \operatorname{Hom}(P, P) \rightarrow \operatorname{Hom}(P, M) \rightarrow 0 .
$$

It is enough to prove that $\operatorname{Hom}(P, F)$ is acyclic. But note that $F$ decomposes as the direct sum of two complexes, namely the complexes

$$
\cdots \rightarrow P_{2} \rightarrow P_{1} \rightarrow Z_{0} P \rightarrow 0 \text { and } 0 \rightarrow P_{-1} \rightarrow P_{-2} \rightarrow \cdots \text {. }
$$


By the observations in the previous paragraph, $\operatorname{Hom}(P, C)$ is acyclic when $C$ is either of these two complexes.

Finally, let us consider (d). Here we consider the map of complexes $Z_{0} \hookrightarrow P$ (where $Z_{0}$ is the complex concentrated entirely in degree 0 , consisting of the zero-cycles of $\left.P, Z_{0} P\right)$. We'll first show that this induces a quasi-isomorphism after applying $\operatorname{Hom}(-, P)$.

Note that there is a short exact sequence of complexes

$$
0 \rightarrow \operatorname{Hom}\left(P / Z_{0}, P\right) \rightarrow \operatorname{Hom}(P, P) \rightarrow \operatorname{Hom}\left(Z_{0}, P\right) \rightarrow 0
$$

and that $P / Z_{0}$ decomposes as the direct sum of

$$
\cdots \rightarrow P_{2} \rightarrow P_{1} \rightarrow 0 \text { and } 0 \rightarrow P_{0} / Z_{0} \rightarrow P_{-1} \rightarrow P_{-2} \rightarrow \cdots .
$$

As in the proof of (c), one argues by the Comparison Theorem that $\operatorname{Hom}(C, P)$ is acyclic when $C$ is either a bounded below complex of projectives or a bounded above acyclic complex. This shows that $\operatorname{Hom}\left(P / Z_{0}, P\right)$ is acyclic, and hence $\operatorname{Hom}(P, P) \rightarrow$ $\operatorname{Hom}\left(Z_{0}, P\right)$ is a quasi-isomorphism.

To complete the proof of (d), just note that our map $\Omega M \rightarrow P$ factors through $Z_{0}$, and that the map $\Omega M \rightarrow Z_{0}$ is a weak equivalence in $\operatorname{Stmod}(T)$. The result then follows from the natural isomorphisms in (b).

Proof of Theorem 3.5 To show that the given functors form a Quillen pair, we check that the right adjoint preserves fibrations and trivial fibrations. The fibrations in both $\operatorname{Stmod}(T)$ and $\operatorname{Mod}-\mathcal{E}_{M}$ are just the surjections. Since each level in $P_{\bullet} M$ is projective, $\operatorname{Hom}\left(P_{\bullet} M,-\right)$ preserves surjections. This functor actually preserves all weak equivalences, as this follows from Lemma 3.6(b). In particular, it preserves trivial fibrations.

Let $L$ and $R$ denote the left and right adjoints in our Quillen pair Mod- $\mathcal{E}_{M} \rightleftarrows$ $\operatorname{Stmod}(T)$. Then $R(M)=\mathcal{E}_{M}$, and we remarked above Lemma 3.6 that $L\left(\mathcal{E}_{M}\right) \cong M$. We also note that $\mathcal{E}_{M}$ is a compact generator for $\operatorname{Ho}\left(\operatorname{Mod}-\mathcal{E}_{M}\right)$, from which it follows by $\left[19\right.$, Section 2.2.2] that the only localizing subcategory of $\operatorname{Ho}\left(\operatorname{Mod}-\mathcal{E}_{M}\right)$ containing $\mathcal{E}_{M}$ is the whole homotopy category itself. (Recall that a localizing subcategory is a full triangulated subcategory that is closed under arbitrary coproducts). A similar statement holds for $\operatorname{Ho}(\operatorname{Stmod}(T))$, using that $M$ is a compact generator for that category.

Let $\underline{L}$ and $\underline{R}$ denote the derived functors of $L$ and $R$. Our task is to show that these give an equivalence of homotopy categories. We first argue that $\underline{R}$ preserves arbitrary coproducts. Let $\left\{N_{\alpha}\right\}$ be a set of $T$-modules. There is of course a natural map $\oplus_{\alpha}\left(R N_{\alpha}\right) \rightarrow R\left(\oplus_{\alpha} N_{\alpha}\right)$. Using that $\mathcal{E}_{M}$ is a generator for $\operatorname{Ho}\left(\operatorname{Mod}-\mathcal{E}_{M}\right)$, it 
follows that this map is an isomorphism if and only if it induces an isomorphism after applying $\left[\mathcal{E}_{M},-\right]_{*}$. But it is easy to check that this is the case, using the adjunctions and the compactness of both $\mathcal{E}_{M}$ and $L\left(\mathcal{E}_{M}\right)$.

Consider the unit and counit of the derived adjunctions

$$
\eta_{X}: X \longrightarrow \underline{R} \underline{L}(X) \text { and } v_{N}: \underline{L} \underline{R}(N) \rightarrow N .
$$

The full subcategory of $\operatorname{Ho}\left(\operatorname{Mod}-\mathcal{E}_{M}\right)$ consisting of all $X$ such that $\eta_{X}$ is an isomorphism is a localizing subcategory - this uses the fact that $R$ preserves coproducts. Likewise, the full subcategory of $\operatorname{Ho}(\operatorname{Stmod}(T))$ consisting of all $N$ such that $v_{N}$ is an isomorphism is a localizing subcategory. To prove that $(\underline{L}, \underline{R})$ gives an equivalence of homotopy categories, it therefore suffices to check that $\eta_{\mathcal{E}_{M}}$ and $v_{M}$ are isomorphisms since $\mathcal{E}_{M}$ and $M$ are generators.

Since $\mathcal{E}_{M}$ is a cofibrant $\mathcal{E}_{M}$-module, $\eta_{\mathcal{E}_{M}}$ is isomorphic in Ho(Mod- $\left.\mathcal{E}_{M}\right)$ to the map $\mathcal{E}_{M} \rightarrow R L\left(\mathcal{E}_{M}\right)$. But this latter map is an isomorphism in Mod- $\mathcal{E}_{M}$.

To check that $v_{M}$ is an isomorphism we need one more step. Note that by Lemma 3.6(c) the map

$$
\mathcal{E}_{M}=\operatorname{Hom}(P \bullet M, P \bullet M) \rightarrow \operatorname{Hom}(P \bullet M, M)=R M
$$

is a quasi-isomorphism. So $\mathcal{E}_{M}$ is a cofibrant-replacement for $R(M)$. Then $v_{M}$ is isomorphic in $\operatorname{Ho}(\operatorname{Stmod}(T))$ to the composite $L\left(\mathcal{E}_{M}\right) \rightarrow L(R M) \rightarrow M$. This is readily seen to be an isomorphism of $T$-modules.

\section{Proof that $\mathcal{M}$ and $\mathcal{M}_{\epsilon}$ are not Quillen equivalent}

In this section we apply the material from the last section to our two stable module categories $\mathcal{M}$ and $\mathcal{M}_{\epsilon}$. We compute the endomorphism dgas of $k$ and $k_{\epsilon}$, and the results of the last section show that $\mathcal{M}$ and $\mathcal{M}_{\epsilon}$ are Quillen equivalent to module categories over these dgas. Finally, we use the results of Dugger-Shipley [7] to prove that these module categories are not Quillen equivalent.

First we claim that both $\mathcal{M}$ and $\mathcal{M}_{\epsilon}$ have compact generators.

Proposition 4.1 The module $\mathbb{Z} / p$ is a compact generator for both $\operatorname{Stmod}(R)$ and $\operatorname{Stmod}\left(R_{\epsilon}\right)$.

Proof First, $\mathbb{Z} / p$ is compact in $\operatorname{Stmod}(R)$ by Lemma 3.2. [19, Lemma 2.2.1] shows that to be a compact generator is equivalent to asking that every localizing subcategory which contains the given compact object is the whole category. 
If a localizing subcategory of $\operatorname{Ho}(\operatorname{Stmod}(R))$ contains $\mathbb{Z} / p$, then it contains $R$ because of the exact sequence $0 \rightarrow \mathbb{Z} / p \rightarrow R \rightarrow \mathbb{Z} / p \rightarrow 0$. So it contains every free module and every $\mathbb{Z} / p$-vector space, and therefore it contains every module by Lemma 2.1 . This shows that $\mathbb{Z} / p$ is a generator of $\operatorname{Stmod}(R)$.

The same proof shows that $\mathbb{Z} / p$ is a compact generator of $\operatorname{Stmod}\left(R_{\epsilon}\right)$.

Next we identify the endomorphism dga of our chosen generator in both cases.

Proposition 4.2 The dga $\mathcal{E}_{k}$ in $\operatorname{Stmod}(R)$ is quasi-isomorphic to the dga $A$ generated over $\mathbb{Z}$ by $e$ and $x$ in degree one and $y$ in degree -1 with the relations $e^{2}=0$, $e x+x e=x^{2}, x y=y x=1$ and the differentials $d e=p, d x=0$, and $d y=0$. That is,

$$
A=\mathbb{Z}\langle e, x, y\rangle /\left(e^{2}=0, e x+x e=x^{2}, x y=y x=1, d e=p, d x=0, d y=0\right)
$$

where $|e|=|x|=1$ and $|y|=-1$.

Proof Let $P$ be the chain complex consisting of $\mathbb{Z} / p^{2}$ in every dimension, where the differential is multiplication by $p$. Note that $P$ is a complete resolution for $k$. Then the dga $\mathcal{E}_{k}$ is quasi-isomorphic to $\operatorname{Hom}(P, P)$. Write $\operatorname{Hom}(P, P)=\operatorname{End}(P)$.

For all $n \in \mathbb{Z}$ we have $\operatorname{End}(P)_{n} \cong \prod_{i \in \mathbb{Z}} \operatorname{Hom}\left(\mathbb{Z} / p^{2}, \mathbb{Z} / p^{2}\right) \cong \prod_{i \in \mathbb{Z}} \mathbb{Z} / p^{2}$. Let $f=\left(f_{i}\right)$ denote an element of $\operatorname{End}(P)_{n}$, where each $f_{i}$ is a map $P_{i} \rightarrow P_{n+i}$. Then the $k$ th entry of $d f$ is the map $p\left(f_{k}+(-1)^{n+1} f_{k-1}\right)$.

Let $1 \in \operatorname{End}(P)_{0}$ denote the tuple where $f_{i}=1$ for all $i$. Let $X \in \operatorname{End}(P)_{1}$ be the tuple where $f_{i}=(-1)^{i}$, and let $Y \in \operatorname{End}(P)_{-1}$ be the tuple where $f_{i}=(-1)^{i+1}$. Note that $X Y=Y X=1$, and $d(X)=d(Y)=0$. Let $E \in \operatorname{End}(P)_{1}$ be the tuple where $f_{i}=1$ if $i$ is even, and $f_{i}=0$ if $i$ is odd. Note that $d(E)=p \cdot 1, E^{2}=0$, and $E X+X E=X^{2}$. This allows us to construct a dga map $A \rightarrow \operatorname{End}(P)$ by sending $x \mapsto X, y \mapsto Y$, and $e \mapsto E$.

We can uniquely write every element of $\operatorname{Hom}\left(\mathbb{Z} / p^{2}, \mathbb{Z} / p^{2}\right)=\mathbb{Z} / p^{2}$ in the form $a+p b$ for $a, b \in\{0, \ldots, p-1\}$. Using this notation, the cycles in $\operatorname{End}(P)_{n}$ for $n$ even are tuples $f$ of the form $f_{i}=a+p b_{i}$, where $a$ is independent of $i$. For $n$ odd the cycles are tuples satisfying $f_{i}=a+p b_{i}$ when $i$ is even, and $f_{i}=(p-a)+p b_{i}$ when $i$ is odd; here again, $a$ is independent of $i$. Independently of the parity of $n$, the boundaries in each degree are tuples where every entry is a multiple of $p$ (that is, tuples satisfying $\left.f_{i}=p b_{i}\right)$. Thus we see that $H_{n}(\operatorname{End}(P)) \cong \mathbb{Z} / p$ for all $n$.

Now, it is easy to verify that in degree $n$ the dga $A$ consists of the free abelian group generated by $x^{n}$ and $e x^{n-1}$. This is valid in negative dimensions as well if 
one interprets $x^{-1}$ as $y$. This description makes it routine to check that our map $A \rightarrow \operatorname{End}(P)$ is a quasi-isomorphism.

Proposition 4.3 The dga $\mathcal{E}_{k_{\epsilon}}$ in $\operatorname{Stmod}\left(R_{\epsilon}\right)$ is quasi-isomorphic to the formal dga $\mathcal{A}_{\epsilon}=\mathbb{Z} / p[x, y] /(x y-1)$ with trivial differential. Here $|x|=1$ and $|y|=-1$.

Proof This time let $P$ be the chain complex with $R_{\epsilon}$ in every dimension, and where the differentials are all multiplication by $\epsilon$. This is a complete resolution of $k$, and so $\mathcal{E}_{k_{\epsilon}}$ is quasi-isomorphic to $\operatorname{End}(P)$.

We again have $\operatorname{End}(P)_{n}=\prod_{i \in \mathbb{Z}} \operatorname{Hom}\left(R_{\epsilon}, R_{\epsilon}\right) \cong \prod_{i \in \mathbb{Z}} R_{\epsilon}$, and we will denote elements by tuples $f=\left(f_{i}\right)$ where $f_{i}: P_{i} \rightarrow P_{n+i}$. Then the $k$ th entry of $d f$ is $\epsilon\left(f_{k}+(-1)^{n+1} f_{k-1}\right)$.

Just as in the previous proof, we define elements $1 \in \operatorname{End}(P)_{0}, X \in \operatorname{End}(P)_{1}$, and $Y \in \operatorname{End}(P)_{-1}$. Note that $d(X)=d(Y)=0, X Y=Y X=1$, but this time we have $p \cdot 1=0$. So we get a map of dgas $\mathcal{A}_{\epsilon} \rightarrow \operatorname{End}(P)$.

Every element in $R_{\epsilon}$ can be written uniquely in the form $a+b \epsilon$ where $a, b \in$ $\{0,1 \ldots, p-1\}$. Repeating the same analysis as in the previous proof, one finds that $H_{n}(\operatorname{End}(P)) \cong \mathbb{Z} / p$ for all $n$, and that $\mathcal{A}_{\epsilon} \rightarrow \operatorname{End}(P)$ is a quasi-isomorphism. $\square$

Corollary 4.4 $\operatorname{Stmod}(R)$ is Quillen equivalent to $\operatorname{Mod}-A$ where $A$ is the dga from Proposition 4.2, and $\operatorname{Stmod}\left(R_{\epsilon}\right)$ is Quillen equivalent to $\operatorname{Mod}-\mathcal{A}_{\epsilon}$ where $\mathcal{A}_{\epsilon}$ is the dga from Proposition 4.3.

Proof This follows from Theorem 3.5 together with Schwede-Shipley [18, Theorem 4.3]; the latter shows that quasi-isomorphic dgas have Quillen equivalent module categories.

Our goal is now the following result.

Theorem 4.5 $\operatorname{Mod}-A$ and $\operatorname{Mod}-\mathcal{A}_{\epsilon}$ are not Quillen equivalent. Hence, $\operatorname{Stmod}(R)$ and $\operatorname{Stmod}\left(R_{\epsilon}\right)$ are not Quillen equivalent either.

The argument can be broken up into the following steps.

(1) If there were a chain of Quillen equivalences between $\operatorname{Mod}-A$ and $\operatorname{Mod}-\mathcal{A}_{\epsilon}$, then the object $A$ would have to be taken to $\mathcal{A}_{\epsilon}$ in the derived equivalence of homotopy categories. This is by Proposition 2.9. 
(2) The categories Mod- $A$ and Mod $-\mathcal{A}_{\epsilon}$ are stable, combinatorial model categories. By [3], any object $X$ in these categories has an associated homotopy endomorphism ring spectrum, denoted $\mathrm{hEnd}(X)$. Then by (1) and [3, Corollary 1.4], it follows that if $\operatorname{Mod}-A$ and $\operatorname{Mod}-\mathcal{A}_{\epsilon}$ were Quillen equivalent then one would have $\mathrm{hEnd}(A) \simeq \mathrm{hEnd}\left(\mathcal{A}_{\epsilon}\right)$ as ring spectra.

(3) The model categories Mod- $A$ and Mod- $\mathcal{A}_{\epsilon}$ are actually $\mathcal{C} h(\mathbb{Z})-$ model categories, meaning that they are tensored, cotensored, and enriched over $\mathcal{C} h(\mathbb{Z})$. They are therefore additive model categories, in the sense of [6]. But [6, Proposition 1.5, Proposition 1.7] then says that that the homotopy endomorphism spectrum for any object in such a category is weakly equivalent to the EilenbergMacLane ring spectrum associated to its endomorphism dga. The endomorphism dga of $A$ is just $A$ itself, and likewise for $\mathcal{A}_{\epsilon}$. So this shows that if Mod- $A$ and Mod $-\mathcal{A}_{\epsilon}$ are Quillen equivalent, then the Eilenberg-MacLane ring spectra corresponding to $A$ and $\mathcal{A}_{\epsilon}$ would be weakly equivalent. That is to say-in the language of [7] $-A$ and $\mathcal{A}_{\epsilon}$ would be topologically equivalent.

By this chain of reasoning, proving Theorem 4.5 reduces to proving that $A$ and $\mathcal{A}_{\epsilon}$ are not topologically equivalent. To get started, we will first prove that $A$ is not quasiisomorphic to $\mathcal{A}_{\epsilon}$. This is not strictly necessary for the rest of our argument, but it sets the stage for the more complicated argument we have to give below.

Proposition 4.6 $A$ is not quasi-isomorphic to $\mathcal{A}_{\epsilon}$.

Proof One way to proceed would be to construct a cofibrant-replacement $Q A \stackrel{\sim}{\longrightarrow} A$ of dgas, and then to show that there is no quasi-isomorphism from $Q A$ to $B$. The obstruction comes from the relation $e x+x e=x^{2}$. While an argument can be made along these lines, we instead give a different proof which will motivate the argument for ring spectra in Proposition 4.7 below.

Note that if $A$ and $\mathcal{A}_{\epsilon}$ were quasi-isomorphic, then there would be an isomorphism between the rings $H_{*}\left(\mathbb{Z} / p \otimes_{\mathbb{Z}}^{L} A\right)$ and $H_{*}\left(\mathbb{Z} / p \otimes_{\mathbb{Z}}^{L} \mathcal{A}_{\epsilon}\right)$. Since $A$ is cofibrant as a module over $\mathbb{Z}$, we have $H_{*}\left(\mathbb{Z} / p \otimes_{\mathbb{Z}}^{L} A\right) \cong H_{*}(\mathbb{Z} / p \otimes A)$, which is the ring

$$
\mathbb{Z} / p\langle e, x, y ; d e=d x=d y=0\rangle /\left(e^{2}=0, e x+x e=x^{2}, x y=y x=1\right)
$$

where $|e|=|x|=1$ and $|y|=-1$. For the other case, we use $C=\mathbb{Z}\langle f ; d f=p\rangle /\left(f^{2}\right)$ as a dga which is weakly equivalent to $\mathbb{Z} / p$ and also cofibrant as a $\mathbb{Z}$-module. We then calculate that

$$
H_{*}\left(\mathbb{Z} / p \otimes_{\mathbb{Z}}^{L} \mathcal{A}_{\epsilon}\right) \cong H_{*}\left(C \otimes \mathcal{A}_{\epsilon}\right) \cong \Lambda_{k}(f) \otimes k[x, y] /(x y-1)
$$


where $|f|=|x|=1$ and $|y|=-1$. It is easy to see that the ring $H_{*}(\mathbb{Z} / p \otimes A)$ is not isomorphic to $H_{*}\left(C \otimes \mathcal{A}_{\epsilon}\right)$-for example, the latter ring is graded-commutative but the former is not. Thus $A$ and $\mathcal{A}_{\epsilon}$ cannot be quasi-isomorphic.

Before proceeding to the next result, we need to recall a few definitions. If $T$ is a ring spectrum, a connective cover for $T$ is a connective ring spectrum $U$ together with a map $U \rightarrow T$ which induces isomorphisms $\pi_{i}(U) \rightarrow \pi_{i}(T)$ for $i \geq 0$. Standard obstruction theory arguments show that connective covers exist, and that any two connective covers are weakly equivalent.

If $T$ is a connective ring spectrum then we can also talk about the Postnikov sections of $T$. The $n$th Postnikov section is a ring spectrum $U$ together with a map $T \rightarrow U$ such that $\pi_{i}(U)=0$ for $i>n$ and $\pi_{i}(T) \rightarrow \pi_{i}(U)$ is an isomorphism for $i \leq n$. Again, a standard obstruction theory argument shows that Postnikov sections exist and are unique up to homotopy - see [5, Section 2.1] for a detailed discussion.

It is easy to see that if $T$ and $T^{\prime}$ are weakly equivalent ring spectra then their connective covers and Postnikov sections are also weakly equivalent ring spectra.

If $B$ is a dga, one can define connective covers and Postnikov sections similarly. It is also possible to give more explicit chain-level models, however. We define the connective cover $C B$ by

$$
[C B]_{i}= \begin{cases}B_{i} & \text { if } i>0, \\ Z_{0} B & \text { if } i=0, \text { and } \\ 0 & \text { if } i<0,\end{cases}
$$

where $Z_{0} B$ denotes the zero-cycles in $B$. Note that there is a map of dgas $C B \rightarrow B$, and this induces isomorphisms in homology in non-negative degrees.

Next define the $n$th Postnikov section of $C B$, denoted by $P_{n}(C B)$ (or just $P_{n}(B)$ by abuse):

$$
\left[P_{n} B\right]_{i}= \begin{cases}C B_{i} & \text { if } i<n, \\ C B_{n} / \operatorname{im}\left(C B_{n+1}\right) & \text { if } i=n, \text { and } \\ 0 & \text { if } i>n .\end{cases}
$$

Again note that there is a map of dgas $C B \rightarrow P_{n} B$. See [7, Section 3.1] for a more thorough discussion of Postnikov sections for dgas.

If $B$ is a dga, let $H B$ denote the Eilenberg-MacLane ring spectrum associated to $B$. It is easy to see that $H(C B)$ is a connective cover for $H B$, and that $H\left(P_{n} B\right)$ is an $n$th Postnikov section for $H(C B)$. 
Proposition 4.7 $A$ and $\mathcal{A}_{\epsilon}$ are not topologically equivalent.

Proof If the two dgas $A$ and $\mathcal{A}_{\epsilon}$ were topologically equivalent then clearly their connective covers and $n$th Postnikov sections of these covers would also be topologically equivalent. We will show here that $P_{2} A$ and $P_{2} \mathcal{A}_{\epsilon}$ are not topologically equivalent. The second Postnikov section of $C \mathcal{A}_{\epsilon}$ is $P_{2} \mathcal{A}_{\epsilon} \cong \mathbb{Z} / p[x] /\left(x^{3}\right)$, where $x$ has degree 1 and $d x=0$. For the second Postnikov section of $C A$ we can use the model

$$
P_{2} A=\mathbb{Z}\langle e, x ; d e=p, d x=0\rangle /\left(e^{2}=0, e x+x e=x^{2}, x^{3}=0\right)
$$

where $e$ and $x$ have degree 1 (this dga clearly has a map from $C A$, and it has the properties of a Postnikov section).

If $P_{2} A$ and $P_{2} \mathcal{A}_{\epsilon}$ were topologically equivalent, then their $H \mathbb{Z} / p$ homology algebras would be isomorphic; that is, we would have an isomorphism of rings between $\pi_{*}\left(H \mathbb{Z} / p \wedge{ }_{S}^{L} H\left(P_{2} A\right)\right)$ and $\pi_{*}\left(H \mathbb{Z} / p \wedge_{S}^{L} H\left(P_{2} \mathcal{A}_{\epsilon}\right)\right)$. We will argue that the latter ring has a nonzero element of degree 1 which commutes (in the graded sense) with every other element of degree 1 , whereas the former ring has no such element.

Since $\mathcal{A}_{\epsilon}$ is a $\mathbb{Z} / p$-algebra, $H\left(P_{2} \mathcal{A}_{\epsilon}\right)$ is an $H \mathbb{Z} / p$-algebra. In particular, the map $H \mathbb{Z} / p \rightarrow H\left(P_{2} \mathcal{A}_{\epsilon}\right)$ is central. It follows that the map

$$
H \mathbb{Z} / p \wedge_{S}^{L} H \mathbb{Z} / p \rightarrow H \mathbb{Z} / p \wedge_{S}^{L} H\left(P_{2} \mathcal{A}_{\epsilon}\right)
$$

is central, and therefore the induced map on homotopy is also central (in the graded sense). If $\mathcal{A}_{*}$ denotes the dual Steenrod algebra $\pi_{*}\left(H \mathbb{Z} / p \wedge{ }_{S}^{L} H \mathbb{Z} / p\right)$, then we are saying we have a central map

$$
\theta: \mathcal{A}_{*} \rightarrow \pi_{*}\left(H \mathbb{Z} / p \wedge_{S}^{L} H\left(P_{2} \mathcal{A}_{\epsilon}\right)\right) .
$$

We claim that $\theta$ is an injection in degree one. To see this, we only need to understand the underlying spectrum of $H\left(P_{2} \mathcal{A}_{\epsilon}\right)$, and as a spectrum it is weakly equivalent to $H \mathbb{Z} / p \vee \Sigma H \mathbb{Z} / p \vee \Sigma^{2} H \mathbb{Z} / p$. The fact that $\theta$ is an injection in degree one then follows at once.

The only thing we need to know here about $\mathcal{A}_{*}$ is that it is graded-commutative and has a nonzero element in degree one ( $\xi_{1}$ for $p=2$ or $\tau_{0}$ for $p$ odd) Milnor [13]. The image of this element under $\theta$ gives us a nonzero central element of the ring $\pi_{*}\left(H \mathbb{Z} / p \wedge_{S}^{L} H\left(P_{2} \mathcal{A}_{\epsilon}\right)\right)$, lying in degree 1. (A little extra work shows that $\pi_{*}\left(H \mathbb{Z} / p \wedge{ }_{S}^{L} H\left(P_{2} \mathcal{A}_{\epsilon}\right)\right) \cong \mathcal{A}_{*}[x] /\left(x^{3}\right)$, but we will not need this $)$.

Our next step is to analyze the graded ring $\pi_{*}\left(H \mathbb{Z} / p \wedge_{S}^{L} H\left(P_{2} A\right)\right)$. The unit map $S \longrightarrow H \mathbb{Z}$ induces an algebra map

$$
\phi: \pi_{*}\left(H \mathbb{Z} / p \wedge_{S}^{L} H\left(P_{2} A\right)\right) \longrightarrow \pi_{*}\left(H \mathbb{Z} / p \wedge_{H \mathbb{Z}}^{L} H\left(P_{2} A\right)\right) .
$$


We claim that $\phi$ is an isomorphism in degree one. To see this we only need to understand $H\left(P_{2} A\right)$ as an $H \mathbb{Z}$-module; and as an $H \mathbb{Z}$-module it is weakly equivalent to $H \mathbb{Z} / p \vee \Sigma H \mathbb{Z} / p \vee \Sigma^{2} H \mathbb{Z} / p$. The fact that $\phi$ is an isomorphism in degree one now follows from the fact that $\mathcal{A}_{*} \rightarrow \pi_{*}\left(H \mathbb{Z} / p \wedge_{H \mathbb{Z}}^{L} H \mathbb{Z} / p\right)$ is an isomorphism in degrees zero and one.

Using what we have just learned about $\phi$, it follows that if $\pi_{*}\left(H \mathbb{Z} / p \wedge{ }_{S}^{L} H\left(P_{2} A\right)\right)$ had a nonzero element of degree one which commutes with all the other elements of degree one, then the same would be true of $\pi_{*}\left(H \mathbb{Z} / p \wedge_{H \mathbb{Z}}^{L} H\left(P_{2} A\right)\right)$. But this latter ring is something which is easy to calculate, because $H \mathbb{Z}$-algebra spectra are modeled by dgas [20]. It is isomorphic to $H_{*}\left(\mathbb{Z} / p \otimes_{\mathbb{Z}}^{L} P_{2} A\right)$, which—since $P_{2} A$ is cofibrant as a $\mathbb{Z}$-module-is the same as

$$
H_{*}\left(\mathbb{Z} / p \otimes_{\mathbb{Z}} P_{2} A\right) \cong \mathbb{Z} / p\langle e, x ; d e=d x=0\rangle /\left(e^{2}=0, e x+x e=x^{2}, x^{3}=0\right) .
$$

An easy check verifies that in this ring there is no nonzero element in degree one which commutes with all others.

Thus, $P_{2} A$ and $P_{2} \mathcal{A}_{\epsilon}$ are not topologically equivalent. We conclude that $A$ and $\mathcal{A}_{\epsilon}$ are not topologically equivalent either.

Proof of Theorem 4.5 This follows immediately from Proposition 4.7 and reductions (1)-(3) made after the statement of the theorem.

Remark 4.8 We could have also approached the proof of Theorem 4.5 by quoting [7, Theorem 7.2]. This result shows that the model categories Mod- $A$ and $\operatorname{Mod}-\mathcal{A}_{\epsilon}$ are Quillen equivalent if and only if there is a cofibrant, compact generator $P \in \operatorname{Mod}-A$ such that $\operatorname{Hom}_{A}(P, P)$ is topologically equivalent to $\mathcal{A}_{\epsilon}$. But such a $P$ would have $[P, P] \cong H_{0}\left(\mathcal{A}_{\epsilon}\right) \cong \mathbb{Z} / p$, and there is only one object in Ho(Mod- $\left.A\right)$ whose set of endomorphisms has exactly $p$ elements-namely, $A$ itself. So we would have $\mathcal{A}_{\epsilon}$ topologically equivalent to $\operatorname{Hom}_{A}(A, A)=A$, and this is contradicted by Proposition 4.7. Remarks (1)-(3) above essentially constitute the proof of [7, Theorem 7.2] in this case.

Recall that dgas are said to be derived equivalent if there is a triangulated equivalence between their homotopy categories of dg-modules. Thus, we have established that $A$ and $\mathcal{A}_{\epsilon}$ are derived equivalent dgas whose model categories of modules are not Quillen equivalent.

Remark 4.9 It is worth noting that $A$ and $\mathcal{A}_{\epsilon}$ are also derived equivalent dgas which, for $p>3$, have non-isomorphic $K$-theories. To see this, recall that Schlichting [17, 
Theorem 1.7] shows that the Waldhausen $K$-theories of the stable module categories of finitely generated modules over $R$ and $R_{\epsilon}$ are not isomorphic at $K_{4}$, provided $p>3$. This is based on the calculations of $K_{3}$ for $R$ and $R_{\epsilon}$ from Evens-Friedlander [8] and [1]. Schlichting actually claims his conclusions for $p$ odd, but the calculations of $K_{3}(\mathbb{Z} / 9)$ in [1] are not correct (see Geisser [9] for the correct answer). Thus we exclude $p=3$ here. Since Schlichting considered the $K$-theory of the cofibrant and compact objects in $\operatorname{Stmod}(R)$ and $\operatorname{Stmod}\left(R_{\epsilon}\right)$, it follows from [4, Corollary 3.10] and Corollary 4.4 that $K(A)$ and $K\left(\mathcal{A}_{\epsilon}\right)$ are not isomorphic for $p>3$.

Remark 4.10 By [3], to every object $X$ in a sufficiently nice, stable, model category one can associate a homotopy endomorphism ring spectrum $\mathrm{hEnd}(X)$. This is an object in the model category of symmetric ring spectra, and such a thing is essentially the same thing as an $A_{\infty}$-ring spectrum. What we have done in this section is to show that these $A_{\infty}$-ring spectra, computed for the object $k$ in each of $\mathcal{M}$ and $\mathcal{M}_{\epsilon}$, are not the same. The proof, however, really doesn't depend on very much of the $A_{\infty}$-structure. Every $A_{\infty}$-ring spectrum has an underlying "homotopy ring spectrum"-that is, a ring object in the homotopy category of spectra - and a careful examination of our arguments shows that these underlying homotopy ring spectra are also different. We do not know, however, how to produce $\mathrm{hEnd}(X)$ as a homotopy ring spectrum without first having it as an actual ring spectrum.

\section{Diagram categories}

Note that $\mathcal{M}$ and $\mathcal{M}_{\epsilon}$ are cofibrantly-generated model categories. So for any small category $I$, there are projective model category structures on the diagram categories $\mathcal{M}^{I}$ and $\mathcal{M}_{\epsilon}^{I}$ where in each case the weak equivalences and fibrations are objectwise. See Hirschhorn [10, Section 11.6]. Our goal in this section is to establish some basic comparisons between the homotopy categories $\operatorname{Ho}\left(\mathcal{M}^{I}\right)$ and $\operatorname{Ho}\left(\mathcal{M}_{\epsilon}^{I}\right)$.

We will need the following lemma. It is well-known, but we include a proof for the reader's convenience.

Lemma 5.1 Let $\mathcal{C}$ be a pointed model category and let $Y$ be a group object in $H o(\mathcal{C})$. For any object $X \in \mathcal{C}$, the two evident abelian group structures on $\operatorname{Ho}(\mathcal{C})(\Sigma X, Y)$ are identical. 
Proof Let $f$ and $g$ be two maps in $\operatorname{Ho}(\mathcal{C})(\Sigma X, Y)$. We consider the diagram

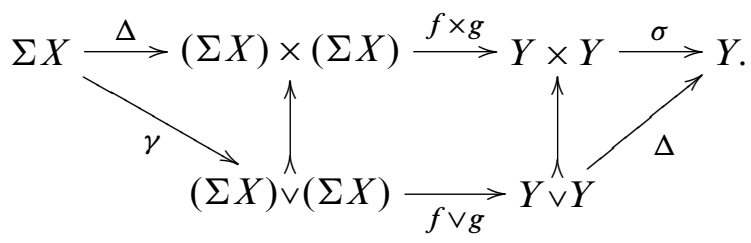

Here $\gamma$ is the comultiplication on $\Sigma X$ constructed by Quillen in [15]. The vertical maps both have the form $(i d, *) \vee(*, i d)$. The top and bottom composites represent the two ways of multiplying $f$ and $g$ in $\operatorname{Ho}(\mathcal{C})(\Sigma X, Y)$.

The properties of a comultiplication ensure that the left triangle commutes, and the properties of a multiplication ensure that the right triangle commutes. The middle square is obviously commutative, so this finishes the proof.

Proposition 5.2 Let $I$ be a small category. Then for any two diagrams $D_{1}, D_{2} \in \mathcal{M}_{\epsilon}^{I}$, the abelian group $\operatorname{Ho}\left(\mathcal{M}_{\epsilon}^{I}\right)\left(D_{1}, D_{2}\right)$ is a $\mathbb{Z} / p$-vector space. For any two diagrams $E_{1}, E_{2} \in \mathcal{M}^{I}$, the abelian group $\operatorname{Ho}\left(\mathcal{M}^{I}\right)\left(E_{1}, E_{2}\right)$ is killed by $p^{2}$.

Proof We give the proof for $\mathcal{M}_{\epsilon}$, and note that the proof for $\mathcal{M}$ is similar.

First note that every diagram $D \in \mathcal{M}_{\epsilon}^{I}$ is an abelian group object, using the objectwise addition $D(i) \oplus D(i) \rightarrow D(i)$. We can therefore study the group structure on $\operatorname{Ho}\left(\mathcal{M}_{\epsilon}^{I}\right)\left(D_{1}, D_{2}\right)$ induced by the target. In this group structure, if $f$ is any map in $\operatorname{Ho}\left(\mathcal{M}_{\epsilon}^{I}\right)\left(D_{1}, D_{2}\right)$ then $n[f]=f+f+\cdots+f(n$ times $)$ is the same as $\left(n\left[\operatorname{id}_{D_{2}}\right]\right) \circ f$.

However, $p\left[\mathrm{id}_{D_{2}}\right]$ is actually equal to the zero map in $\mathcal{M}_{\epsilon}^{I}$ (even before going to the homotopy category). So $p[f]$ is also zero.

It is natural to wonder whether there exists a small category $I$ and diagrams $D_{1}, D_{2}: I \rightarrow$ $\mathcal{M}$ such that $\operatorname{Ho}\left(\mathcal{M}^{I}\right)\left(D_{1}, D_{2}\right)$ is not a $\mathbb{Z} / p$-vector space. So far we have not been able to find such examples. We'll next describe a result showing that for simple categories $I$ such examples do not exist.

A direct Reedy category is a category $I$ in which every object can be assigned a non-negative integer (called its degree) such that every non-identity morphism raises degree [10, Definition 15.1.2]. This is a special case of the more general notion of Reedy category.

If $I$ is a Reedy category and $\mathcal{C}$ is a model category, then there is a Reedy model structure on $\mathcal{C}^{I}$, defined in [10, Section 15.3]. The weak equivalences are the objectwise weak equivalences, and when $\mathcal{C}$ is cofibrantly-generated this model structure is Quillen 
equivalent to the projective model structure on $\mathcal{C}^{I}$. When $I$ is a direct Reedy category then the Reedy fibrations are precisely the objectwise fibrations, and so the Reedy and projective model structures on $\mathcal{C}^{I}$ coincide. The upshot is that this gives us a nice description of the projective cofibrations in $\mathcal{C}^{I}$ : they are the Reedy cofibrations of [10, Definition 15.3.2].

Proposition 5.3 Let $I$ be a small, direct Reedy category. Then for any two diagrams $D_{1}, D_{2} \in \mathcal{M}^{I}$, the abelian group $\operatorname{Ho}\left(\mathcal{M}^{I}\right)\left(D_{1}, D_{2}\right)$ is a $\mathbb{Z} / p$-vector space.

By the same proof as for Proposition 5.2, the result reduces to proving that for any diagram $D \in \mathcal{M}^{I}$ the map $p\left[\operatorname{id}_{D}\right]$ represents zero in $\operatorname{Ho}\left(\mathcal{M}^{I}\right)(D, D)$. We will prove this using a few lemmas.

Lemma 5.4 Let $A \succ B$ be a cofibration in $\mathcal{M}$ and let $F \rightarrow B$ be a surjection where $F$ is a free module. Then any commutative square

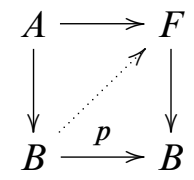

(where the bottom map is multiplication-by- $p$ ) has a lifting as shown.

Proof One first verifies the lemma for the generating cofibrations, which are $0 \rightarrow k$, $0 \rightarrow R$, and $k \rightarrow R$. The first two cases are immediate, and the third is an easy exercise.

Now use that every monomorphism in $\mathcal{M}$ is a direct sum of monomorphisms of type $0 \rightarrow k, 0 \rightarrow R$, id: $k \rightarrow k$, id: $R \rightarrow R$, and $k \hookrightarrow R$, by Proposition 2.6.

Proposition 5.5 Let $I$ be a small, direct Reedy category. For any diagram $D \in \mathcal{M}^{I}$, the map $p\left[\operatorname{id}_{D}\right]: D \rightarrow D$ is null-homotopic in $\mathcal{M}^{I}$.

Proof Notice that we may as well assume that $D$ is Reedy cofibrant in $\mathcal{M}^{I}$. Choose a diagram of free modules $F$ and a surjection $F \rightarrow D$ (that is to say, factor the map $0 \rightarrow D$ as a trivial cofibration followed by a fibration). We will show that the map $p: D \rightarrow D$ factors through $F$.

Choose a degree function on $I$. For each $i \in I$ of degree 0 , choose a factorization of $p: D_{i} \rightarrow D_{i}$ through $F_{i}$; such a factorization exists by the above lemma applied with $A \rightarrow B$ being $0 \rightarrow D_{i}$.

We may assume by induction that we have a partial map of diagrams $D \rightarrow F$ defined on the subdiagrams indexed by elements in $I$ of degree less than $n$. By [10, Discussion 
at the end of Section 15.2], to extend this to the subdiagrams indexed by elements of degree less than $n+1$ we must choose, for every object $i \in I$ of degree $n$, a lifting in the diagram

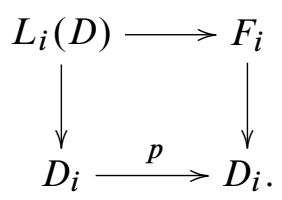

Here $L_{i}(D)$ is the latching object of $D$ at $i$, and we have implicitly used that the matching objects of $D$ and $F$ are all trivial because $I$ is a direct Reedy category.

Since $D$ is Reedy cofibrant, the maps $L_{i}(D) \rightarrow D_{i}$ are all cofibrations. So liftings in the above square exist by Lemma 5.4, and we are done.

Proof of Proposition 5.3 Immediate from Proposition 5.5.

\section{A spectral sequence for mapping spaces}

In this section we continue our comparison of $\operatorname{Ho}\left(\mathcal{M}^{I}\right)$ and $\operatorname{Ho}\left(\mathcal{M}_{\epsilon}^{I}\right)$ when $I$ is a relatively simple indexing category. We are able to give some results in situations where the $\mathbb{Z} / p$-cohomological dimension of $I$ (defined below) is less than or equal to 1 .

\subsection{Background}

We begin with some homological algebra. Let $\mathcal{V}$ denote the category of vector spaces over a field $F$, and let $I$ be a small category. Then the category of diagrams $\mathcal{V}^{I}$ is an abelian category with enough projectives and injectives. So given diagrams $A, B \in \mathcal{V}^{I}$, one has groups $\operatorname{Ext}_{\mathcal{V}^{I}}^{n}(A, B)$ defined in the usual way via resolutions.

It will be convenient for us to know a little about projectives in $\mathcal{V}^{I}$. For each $i \in I$, let $F_{i}: I \rightarrow \mathcal{S} e t$ denote the free diagram generated at $i$; that is, $F_{i}(j)=I(i, j)$ for all $j \in I$. If $X \in \mathcal{V}$, let $F_{i} \otimes X \in \mathcal{V}^{I}$ denote the diagram defined by

$$
\left(F_{i} \otimes X\right)(j)=I(i, j) \otimes X=\coprod_{I(i, j)} X .
$$

We will sometimes write $F_{i}(X)$ in place of $F_{i} \otimes X$.

Note that for each $i \in I$ one has adjoint functors

$$
F_{i}(-): \mathcal{V} \rightleftarrows \mathcal{V}^{I}: \mathrm{Ev}_{i}
$$


where the right adjoint sends a diagram to its value at $i$. It follows that for each object $X \in \mathcal{V}$ and each $i \in I$, the diagram $F_{i}(X)$ is projective in $\mathcal{V}^{I}$.

Let $A \in \mathcal{V}^{I}$. One can show that $A$ has a canonical projective resolution obtained by normalizing the evident simplicial object

$$
\bigoplus_{i_{0}} F_{i_{0}}\left[A\left(i_{0}\right)\right] \gtreqless \bigoplus_{i_{0} \rightarrow i_{1}} F_{i_{1}}\left[A\left(i_{0}\right)\right] \gtreqless \bigoplus_{i_{0} \rightarrow i_{1} \rightarrow i_{2}} F_{i_{2}}\left[A\left(i_{0}\right)\right] \leftleftarrows
$$

This is a kind of bar resolution. Applying $\operatorname{Hom}_{\mathcal{V}^{I}}(-, B)$ and using the apparent adjunctions, it follows that the groups $\operatorname{Ext}^{n}(A, B)$ can be computed as the cohomology groups of the cochain complex associated to the cosimplicial abelian group

$$
\prod_{i_{0}} \mathcal{V}\left(A\left(i_{0}\right), B\left(i_{0}\right)\right) \Longrightarrow \prod_{i_{0} \rightarrow i_{1}} \mathcal{V}\left(A\left(i_{0}\right), B\left(i_{1}\right)\right) \Longrightarrow \prod_{i_{0} \rightarrow i_{1} \rightarrow i_{2}} \mathcal{V}\left(A\left(i_{0}\right), B\left(i_{2}\right)\right) \equiv
$$

We'll call this complex $\mathcal{B}_{(\mathcal{V}, I)}(A, B)$.

We define the $\boldsymbol{F}$-cohomological dimension of $I$ to be the smallest integer $n$ with the property that $\operatorname{Ext}^{n+1}(A, B)=0$ for all $A, B \in \mathcal{V}^{I}$.

Example 6.2 Let $G$ be a group, regarded as a category with one object. Then an element of $\mathcal{V}^{G}$ is just a representation of $G$, and we are dealing with the usual homological algebra of representations. So for instance the group $G=\mathbb{Z} / 2$ has cohomological dimension equal to $\infty$ over the field $\mathbb{F}_{2}$, because $\operatorname{Ext}^{n}(R, R) \neq 0$ for all $n$ where $R$ denotes the trivial representation of $G$ on $\mathbb{F}_{2}$. The cohomological dimension over $\mathbb{Q}$ is equal to zero.

Example 6.3 If $G$ is a directed graph on a set $S$, one may speak of the free category $\mathcal{F} G$ generated by $G$. This is the category with object set equal to $S$, and whose morphisms are formal compositions of the edges in $G$. In the algebra literature $G$ is called a quiver, and a diagram in $\mathcal{V}^{\mathcal{F} G}$ is called a representation of this quiver. It is known that the free categories $\mathcal{F} G$ have $F$-cohomological dimension less than or equal to 1 , for every field $F$.

For each $n$, let $[n]$ denote the usual category of $n$-composable maps $0 \rightarrow 1 \rightarrow$ $\cdots \rightarrow n$. This is the free category generated by the evident directed graph, and so its cohomological dimension is less than or equal to 1 . An easy computation shows that it is actually equal to 1 .

Example 6.4 Let $I$ be the "coequalizer" category consisting of three objects

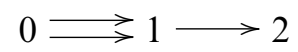


and four non-identity maps: the three shown above, and the map which is equal to the two composites. There are three basic projectives, namely $F_{0}(k), F_{1}(k)$, and $F_{2}(k)$. These are the diagrams

$$
k \rightrightarrows k \oplus k \rightarrow k, \quad 0 \rightrightarrows k \stackrel{=}{\longrightarrow} k, \quad \text { and } \quad 0 \rightrightarrows 0 \rightarrow k .
$$

In the first diagram the two maps $k \rightarrow k \oplus k$ are the two canonical inclusions into the direct sum; the map $k \oplus k \rightarrow k$ is the coequalizer.

Any diagram of the form $[0 \rightrightarrows 0 \rightarrow V]$ is projective; it is $F_{2}(V)$. Any diagram of the form [0 $\rightrightarrows V \rightarrow 0$ ] has a projective resolution of length one: namely, the resolution $0 \rightarrow$ $F_{2}(V) \rightarrow F_{1}(V) \rightarrow 0$. Finally, any diagram [ $V \rightrightarrows 0 \rightarrow 0$ ] has a projective resolution of length two: the resolution has the form $0 \rightarrow F_{2}(V) \rightarrow F_{1}(V \oplus V) \rightarrow F_{0}(V) \rightarrow 0$.

Note that any diagram $\left[V_{0} \rightrightarrows V_{1} \rightarrow V_{2}\right]$ may be built via successive extensions of the three types of diagrams considered in the last paragraph. Namely, one has short exact sequences

$$
\begin{gathered}
0 \rightarrow\left[0 \rightrightarrows 0 \rightarrow V_{2}\right] \rightarrow\left[V_{0} \rightrightarrows V_{1} \rightarrow V_{2}\right] \rightarrow\left[V_{0} \rightrightarrows V_{1} \rightarrow 0\right] \rightarrow 0 \\
\text { and } 0 \rightarrow\left[0 \rightrightarrows V_{1} \rightarrow 0\right] \rightarrow\left[V_{0} \rightrightarrows V_{1} \rightarrow 0\right] \rightarrow\left[V_{0} \rightrightarrows 0 \rightarrow 0\right] \rightarrow 0 .
\end{gathered}
$$

It follows easily that $\operatorname{Ext}^{n}(D, E)=0$ for any $n>2$ and any diagrams $D, E \in \mathcal{V}^{I}$.

A simple computation shows that if $D=[k \rightrightarrows 0 \rightarrow 0]$ and $E=[0 \rightrightarrows 0 \rightarrow k]$ then $\operatorname{Ext}^{2}(D, E)=k$. So the cohomological dimension of $I$ is equal to 2 .

\subsection{The spectral sequence}

Now we return to our model categories $\mathcal{M}$ and $\mathcal{M}_{\epsilon}$. If $X \in \mathcal{M}$, we again let $F_{i} \otimes X \in \mathcal{M}^{I}$ denote the diagram defined by

$$
\left(F_{i} \otimes X\right)(j)=I(i, j) \otimes X=\coprod_{I(i, j)} X .
$$

Note that for each $i \in I$ one has a Quillen adjunction

$$
F_{i} \otimes(-): \mathcal{M} \rightleftarrows \mathcal{M}^{I}: \mathrm{Ev}_{i}
$$

where the right adjoint sends a diagram to its value at $i$. Consequently, for any diagram $E \in \mathcal{M}^{I}$ there is a natural weak equivalence of mapping spaces

$$
\underline{\mathcal{M}^{I}}\left(F_{i} \otimes X, E\right) \simeq \underline{\mathcal{M}}(X, E(i)) .
$$


Let $D \in \mathcal{M}^{I}$. One can form the following simplicial object:

$$
\coprod_{i_{0}} F_{i_{0}} \otimes D\left(i_{0}\right) \longleftarrow \coprod_{i_{0} \rightarrow i_{1}} F_{i_{1}} \otimes D\left(i_{0}\right) \gtreqless \coprod_{i_{0} \rightarrow i_{1} \rightarrow i_{2}} F_{i_{2}} \otimes D\left(i_{0}\right) \leftleftarrows
$$

One can show that the homotopy colimit of this simplicial diagram is weakly equivalent to $D$. It follows that for any fibrant diagram $E \in \mathcal{M}^{I}$, the mapping space $\underline{\mathcal{M}^{I}}(D, E)$ is the homotopy limit of a corresponding cosimplicial diagram of mapping spaces. Using our adjunctions mentioned above, we have that $\underline{\mathcal{M}^{I}}(D, E)$ is weakly equivalent to the homotopy limit of the cosimplicial simplicial set

$$
\prod_{i_{0}} \underline{\mathcal{M}}\left(D\left(i_{0}\right), E\left(i_{0}\right)\right) \Longrightarrow \prod_{i_{0} \rightarrow i_{1}} \underline{\mathcal{M}}\left(D\left(i_{0}\right), E\left(i_{1}\right)\right) \Longrightarrow \prod_{i_{0} \rightarrow i_{1} \rightarrow i_{2}} \underline{\mathcal{M}}\left(D\left(i_{0}\right), E\left(i_{2}\right)\right) \cdots
$$

Call this cosimplicial simplicial set $\underline{\mathcal{B}}(D, E)$. There is a resulting spectral sequence for computing the homotopy groups of the space $\underline{\mathcal{M}^{I}}(D, E)$.

Note that each mapping space $\underline{\mathcal{M}}(X, Y)$ is naturally a simplicial abelian group, so using the Dold-Kan equivalence the above cosimplicial simplicial set can be turned into a double chain complex. The spectral sequence in question is just the usual spectral sequence for a double complex.

Our next task is to identify the $E_{2}$-term of the spectral sequence. This is the cohomology of the cochain complexes obtained by applying $\pi_{q}$ to each object in $\underline{\mathcal{B}}(D, E)$. But note that $\pi_{q} \mathcal{M}(X, Y) \cong \operatorname{Ho}(\mathcal{M})\left(\Sigma^{q} X, Y\right)$. One finds that this cochain complex can be identified with $\mathcal{B}_{(\mathcal{V}, I)}\left(\Sigma^{q} D, E\right)$ where $\mathcal{V}=\operatorname{Ho}(\mathcal{M})$ and we regard $\Sigma^{q} D$ and $E$ as diagrams $\Sigma^{q} D: I \rightarrow \operatorname{Ho}(\mathcal{M})$ and $E: I \rightarrow \operatorname{Ho}(\mathcal{M})$.

Putting everything together, we find that our spectral sequence has

$$
E_{2}^{p, q}=\operatorname{Ext}_{\mathcal{V}^{I}}^{p}\left(\Sigma^{q} D, E\right) \Rightarrow \pi_{q-p}\left[\underline{\mathcal{M}^{I}}(D, E)\right] .
$$

With this indexing the differential $d_{r}$ is a map $d_{r}: E_{r}^{p, q} \rightarrow E_{r}^{p+r, q+r-1}$. Note that if the $\mathbb{Z} / p$-cohomological dimension of $I$ is less than or equal to 1 , then the $E_{2}$-term is concentrated in two adjacent columns and the spectral sequence collapses.

Remark 6.6 Everything that we've said above applies equally well to the model category $\mathcal{M}_{\epsilon}$. If $D$ and $E$ are diagrams in $\mathcal{M}_{\epsilon}^{I}$, one obtains a corresponding spectral sequence

$$
E_{2}^{p, q}=\operatorname{Ext}_{\mathcal{V}^{I}}^{p}\left(\Sigma^{q} D, E\right) \Rightarrow \pi_{q-p}\left[\underline{\mathcal{M}_{\epsilon}^{I}}(D, E)\right] .
$$

If $D$ and $E$ are diagrams in $\operatorname{Vect}^{I}$ then we can regard them as lying both in $\mathcal{M}^{I}$ and $\mathcal{M}_{\epsilon}^{I}$, and so we can examine both spectral sequences at once. They have the same $E_{2}$-terms, but may have different differentials. 


\subsection{An application}

The functors Vect $\stackrel{j}{\longrightarrow} \mathcal{M} \stackrel{\Gamma}{\longrightarrow}$ Vect induce functors

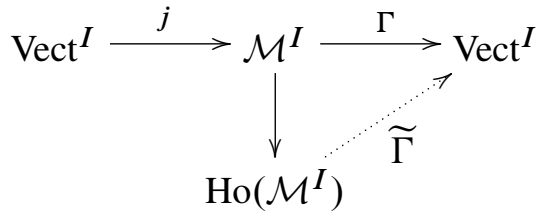

where the existence of $\widetilde{\Gamma}$ follows from the fact that $\Gamma$ takes objectwise weak equivalences to isomorphisms. As $\Gamma \circ j=\mathrm{id}$, we have

$$
\operatorname{Vect}^{I} \hookrightarrow \operatorname{Ho}\left(\mathcal{M}^{I}\right) \rightarrow \operatorname{Vect}^{I} .
$$

Proposition 6.8 If the $\mathbb{Z} / p$-cohomological dimension of $I$ is less than or equal to one, then $j: \operatorname{Vect}^{I} \rightarrow \operatorname{Ho}\left(\mathcal{M}^{I}\right)$ is surjective on isomorphism classes. Said differently, every diagram $D \in \mathcal{M}^{I}$ is weakly equivalent to $j \Gamma(D)$.

The same thing holds with $\mathcal{M}$ replaced by $\mathcal{M}_{\epsilon}$.

Proof We can assume $D$ is a cofibrant diagram. Since $j$ : Vect $\rightarrow \operatorname{Ho}(\mathcal{M})$ is an equivalence of categories, so is the induced map $\operatorname{Vect}^{I} \rightarrow \operatorname{Ho}(\mathcal{M})^{I}$. So there exists a diagram $E \in \operatorname{Vect}^{I}$ such that $D$ and $E$ are isomorphic when regarded as diagrams in $\operatorname{Ho}(\mathcal{M})^{I}$. The rest of the proof will use obstruction theory to produce a weak equivalence $D \rightarrow E$.

Start by choosing a framing for the diagram $D: I \rightarrow \mathcal{M}$. If $c \mathcal{M}$ denotes the category of cosimplicial objects over $\mathcal{M}$, such a framing is a functor $\widetilde{D}: I \rightarrow c \mathcal{M}$ taking its values in the Reedy cofibrant objects, together with a natural isomorphism $\widetilde{D}^{0} \rightarrow D$ (we can insist on an isomorphism here because all objects of $\mathcal{M}$ are cofibrant); see [11, Chapter 5]. Consider the following double chain complex of abelian groups:

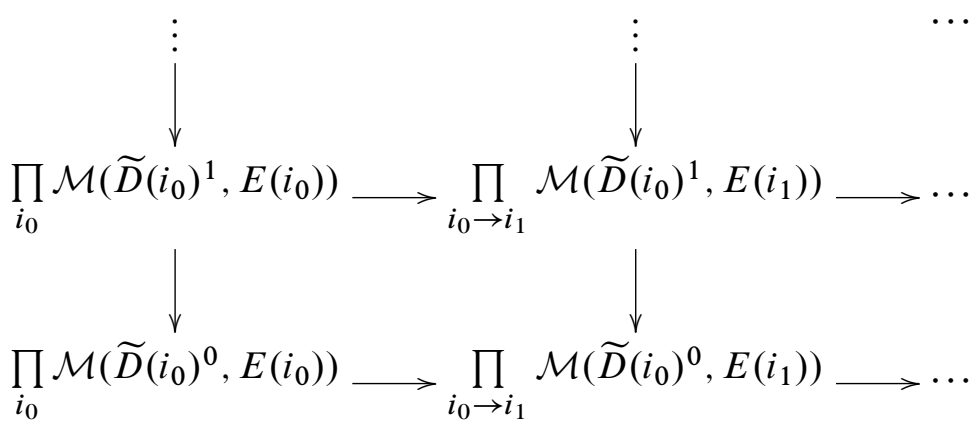


The spectral sequence of (6.5) coincides with the spectral sequence for this double complex where one first takes homology in the vertical direction and then in the horizontal direction.

We know that $D$ and $E$ are isomorphic when regarded as diagrams $I \rightarrow \operatorname{Ho}(\mathcal{M})$. Let $\alpha$ be such an isomorphism. For each $i \in I$, choose a weak equivalence $f_{i}: D(i) \rightarrow E(i)$ representing $\alpha_{i}$ (we know such a weak equivalence exists because $D(i)$ is cofibrant and $E(i)$ is fibrant). The collection of all these $f_{i}$ represents an element $z$ in the lower left group in the above double complex. Our goal is to produce an element in $H_{0}(-)$ of the total complex which has $z$ as its first component, because this will then represent an element of $\pi_{0} \mathcal{M}^{I}(\mathcal{D}, E)$.

The $f_{i}$ do not exactly give a map of diagrams from $D$ to $E$, but they give a "homotopy commutative" map of diagrams. If $z_{1}$ denotes the image of $z$ under the horizontal differential in the double complex, this precisely says that $z_{1}$ is the image of some element $z_{2}$ under the vertical differential. That is, for every map $c: i \rightarrow j$ in $I$ we can choose a homotopy between the composites $f_{j} \circ D(c)$ and $E(c) \circ f_{i}$.

The pair $\left(z, z_{2}\right)$ constitutes the beginning of a 0 -cycle in the total complex. There are obstructions to extending it further, but the fact that the spectral sequence for our double complex is concentrated along the first two columns-because of our assumption on the cohomological dimension of $I$-shows precisely that all these obstructions vanish. So we can construct our desired $0-$ cycle, and the proof is complete.

Corollary 6.9 Suppose the $\mathbb{Z} / p$-cohomological dimension of $I$ is less than or equal to one. Then every abelian group $\operatorname{Ho}\left(\mathcal{M}^{I}\right)(A, B)$ is a $\mathbb{Z} / p$-vector space.

Proof Let $A, B \in \mathcal{M}^{I}$. By Proposition $6.8, B$ is weakly equivalent to a diagram $D$ of $k$-vector spaces. So $\operatorname{Ho}\left(\mathcal{M}^{I}\right)(A, B) \cong \operatorname{Ho}\left(\mathcal{M}^{I}\right)(A, D)$. But the identity map id: $D \rightarrow D$ is $p$-torsion, and so by arguments used in the proof of Proposition 5.2 it follows that every element of $\operatorname{Ho}\left(\mathcal{M}^{I}\right)(A, D)$ is $p$-torsion as well.

Proposition 6.10 Suppose the $\mathbb{Z} / p$-cohomological dimension of $I$ is less than or equal to one. Then the functors $\operatorname{Vect}^{I} \rightarrow \operatorname{Ho}\left(\mathcal{M}^{I}\right)$ and $\operatorname{Vect}^{I} \rightarrow \operatorname{Ho}\left(\mathcal{M}_{\epsilon}^{I}\right)$ are both bijections on isomorphism classes. For every two diagrams $A, B \in \mathrm{Vect}^{I}$, the abelian groups $\operatorname{Ho}\left(\mathcal{M}^{I}\right)(A, B)$ and $\operatorname{Ho}\left(\mathcal{M}_{\epsilon}^{I}\right)(A, B)$ are isomorphic.

Proof The statement that the $j$ functors are bijections on isomorphism classes follows from Proposition 6.8 together with the remarks made immediately prior to it. For the second statement, consider the two spectral sequences

$$
E_{2}^{p, q}=\operatorname{Ext}_{\mathcal{V}^{I}}^{p}\left(\Sigma^{q} A, B\right) \Rightarrow \pi_{q-p}\left[\underline{\mathcal{M}^{I}}(D, E)\right]
$$


and

$$
E_{2}^{p, q}=\operatorname{Ext}_{\mathcal{V}^{I}}^{p}\left(\Sigma^{q} A, B\right) \Rightarrow \pi_{q-p}\left[\underline{\mathcal{M}_{\epsilon}^{I}}(D, E)\right] .
$$

Both spectral sequences are concentrated along the columns $p=0$ and $p=1$, due to the assumption on the cohomological dimension of $I$. So both spectral sequences collapse. Since $\operatorname{Ho}\left(\mathcal{M}^{I}\right)(A, B)$ and $\operatorname{Ho}\left(\mathcal{M}_{\epsilon}^{I}\right)(A, B)$ are both $\mathbb{Z} / p$-vector spaces, there are no extension problems when passing from the $E_{\infty}$ terms. The result now follows from the fact that the $E_{2}$-terms of the two spectral sequences are identical. $\square$

\section{References}

[1] J E Aisbett, E Lluis-Puebla, V Snaith, On $K_{*}(\mathbf{Z} / n)$ and $K_{*}\left(\mathbf{F}_{q}[t] /\left(t^{2}\right)\right)$, Mem. Amer. Math. Soc. 57, 329 (1985) vi+200 MR803974 With an appendix by Christophe Soulé

[2] H-J Baues, F Muro, Cohomologically triangulated categories. I, J. K-Theory 1 (2008) 3-48 MR2424565

[3] D Dugger, Spectral enrichments of model categories, Homology, Homotopy Appl. 8 (2006) 1-30 MR2205213

[4] D Dugger, B Shipley, K-theory and derived equivalences, Duke Math. J. 124 (2004) 587-617 MR2085176

[5] D Dugger, B Shipley, Postnikov extensions of ring spectra, Algebr. Geom. Topol. 6 (2006) 1785-1829 MR2263050

[6] D Dugger, B Shipley, Enriched model categories and an application to additive endomorphism spectra, Theory Appl. Categ. 18 (2007) No. 15, 400-439 MR2342167

[7] D Dugger, B Shipley, Topological equivalences for differential graded algebras, Adv. Math. 212 (2007) 37-61 MR2319762

[8] L Evens, E M Friedlander, On $\left(K_{*}\left(\mathbf{Z} / p^{2} \mathbf{Z}\right)\right.$ and related homology groups, Trans. Amer. Math. Soc. 270 (1982) 1-46 MR642328

[9] T Geisser, On $K_{3}$ of Witt vectors of length two over finite fields, $K$-Theory 12 (1997) 193-226 MR1480590

[10] P S Hirschhorn, Model categories and their localizations, Mathematical Surveys and Monographs 99, Amer. Math. Soc. (2003) MR1944041

[11] M Hovey, Model categories, Mathematical Surveys and Monographs 63, Amer. Math. Soc. (1999) MR1650134

[12] B Keller, Deriving DG categories, Ann. Sci. École Norm. Sup. (4) 27 (1994) 63-102 MR1258406

[13] J Milnor, The Steenrod algebra and its dual, Ann. of Math. (2) 67 (1958) 150-171 MR0099653 
[14] F Muro, Triangulated categories with universal Toda bracket Available at http:// www.maths.leeds.ac.uk/pure/algebra/TriCatSlides/TriCatSlides.html

[15] D G Quillen, Homotopical algebra, Lecture Notes in Mathematics 43, Springer, Berlin (1967) MR0223432

[16] O Renaudin, Théories homotoiques de Quillen combinatoires et dérivateurs de Grothendieck, preprint (2006) arXiv:math.AT/0603339

[17] M Schlichting, A note on K-theory and triangulated categories, Invent. Math. 150 (2002) 111-116 MR1930883

[18] S Schwede, B Shipley, Algebras and modules in monoidal model categories, Proc. London Math. Soc. (3) 80 (2000) 491-511 MR1734325

[19] S Schwede, B Shipley, Stable model categories are categories of modules, Topology 42 (2003) 103-153 MR1928647

[20] B Shipley, HZZ-algebra spectra are differential graded algebras, Amer. J. Math. 129 (2007) 351-379 MR2306038

[21] B Toën, G Vezzosi, A remark on $K$-theory and $S$-categories, Topology 43 (2004) 765-791 MR2061207

University of Oregon, Department of Mathematics Eugene, OR 97403, USA

Department of Mathematics, Statistics and Computer Science 508 SEO (m/c 249), 851 S. Morgan St., Chicago, IL 60607-7045, USA

ddugger@uoregon.edu, bshipley@math.uic.edu http: //www.math.uic.edu/ bshipley/

Received: 12 June 2008 Revised: 17 December 2008 